Revue d'histoire de l'Amérique française

RAS REVUE D.HISTOIRE DE L'AMÉRIQUE FRANÇAISE

\title{
Un dossier de plantation de Saint-Domingue (1745-1829) (suite)
}

\section{Roger Massio}

Volume 6, numéro 1, juin 1952

URI : https://id.erudit.org/iderudit/301504ar

DOI : https://doi.org/10.7202/301504ar

Aller au sommaire du numéro

Éditeur(s)

Institut d'histoire de l'Amérique française

ISSN

0035-2357 (imprimé)

1492-1383 (numérique)

Découvrir la revue

Citer cet article

Massio, R. (1952). Un dossier de plantation de Saint-Domingue (1745-1829)

(suite). Revue d'histoire de l'Amérique française, 6(1), 62-109.

https://doi.org/10.7202/301504ar d'utilisation que vous pouvez consulter en ligne.

https://apropos.erudit.org/fr/usagers/politique-dutilisation/ 


\section{UN DOSSIER DE PLANTATION DE SAINT-DOMINGUE (1745-1829) \\ (suite)}

Chapitre QUATRIÈmE

ETAT DE L'ESPRIT PUBLIC A SAINT-DOMINGUE, SOUS LA REVOLUTION

\section{LA SITUATION POLITIQUE \\ D'APR ES LA CORRESPONDANCE DES PROCUREURS, LES FRERES TERRIEN (1790)}

Les Terrien, procureurs de l'habitation Navailles, étaient originaires de Nantes ${ }^{58}$. Ils avaient une maison de commerce à SaintMarc. Ils ne jouèrent pas un grand rôle politique pendant la Révolution; on ne les voit mêlés à aucun des grands débats. L'aîné sera seulement député suppléant de Saint-Marc à la deuxième assemblée coloniale qui se réunit au Cap, d'août 1791 à la fin de $1792^{59}$.

Mais de Saint-Marc, ils seront bien placés pour observer les événements généraux et leurs lettres à Navailles-Bonnas dépassant

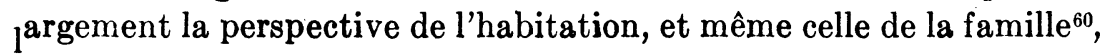

58. Les frères Terrien étaient originaires de Nantes. Une autre branche de Terrien était originaire de La Chapelle-sur-Erdre; mais les Terrien, originaires de La Chapelle-sur-Erdre, n'ont que des rapports lointains avec nos Terrien, orizinaires de Nantes. (Renseignements obligeamment procurés par $M$. le Vicomte de $P$. de Freslon). A 286.

59. Journal des Colonies no II - s.d. [ juillet 1791 ], 9- Arch. nat. A.Dxx.

69. Les documents dépassent trop largement la perspective familiale ou individuelle pour qu'on puisse faire des coupures. Mieux vaut donner les documents dans leur coulée entière. On a tout le vrai et le vif des situations. 
seront comme une large chronique du quartier et même de la colonie, une sorte de journal politique. Il n'est qu'un regret, c'est que nous n'ayons retrouvé que leurs lettres de 1790 .

L'objectivité de ces lettres est évidemment toute relative. Les Terrien sont des blancs qui administrent de grandes plantations. Leur position politique est très nette. Il n'est pas besoin qu'ils s'expliquent très longuement ni très ouvertement sur le fond des problèmes sociaux, c'est-à-dire d'abord sur le problème des gens de couleur, pour qu'on s'en rende compte. Et leur point de vue ne changera pas: ils soutiendront la cause des colons blancs. Mais aussi ils sont négociants, des hommes ouverts, par moment, à des vues plus larges.

De plus, ces lettres sont très curieuses du point de vue du style. Les Terrien écrivaient eux-mêmes leurs lettres et ils les écrivaient bien. Toute l'éducation du XVIIIème siècle, dans la bourgeoisie non appelée à des tâches spéciales, consistait dans la préparation à la tenue des correspondances.

La première lettre est du 6 mai 1790. Navailles a quitté Larreule et se trouve alors à Bordeaux, à l'Hôtel Seguineau. Depuis septembre 1789, il s'est fait admettre au club des colons des îles qui s'est constitué sous la direction de David-Gradis pour la défense générale de la classe des planteurs ${ }^{61}$. Ce club est en relations suivies avec la société correspondante des colons américains qui a son siège à Paris, à l'Hôtel Massiac ${ }^{62}$. Comme beaucoup de propriétaires coloniaux, il est inquiet ou on l'a rendu inquiet de ce qui allait se passer à la colonie. Depuis plus d'un an, les partisans de l'abolition de l'esclavage attaquent le commerce négrier qui assure la maind'œuvre des îles. Or s'en prendre à la traite, c'est s'en prendre à l'origine de l'esclavage. "La menace de voir bouleverser le régime de l'esclavage que tout le monde aux colonies considérait comme la base même de la vie sociale ${ }^{63}$," était le grand sujet des préoccupations de Navailles si nous en croyons la lettre qui lui est adressée le 14 janvier 1790 au moment où il quitte Larreule pour Bordeaux.

61. Arch. Nat. Dxxv 86.

62. Club Massiac - Groupement dès le 20 aout 1789 d'un certain nombre d'entre les grands propriétaires coloniaux résidant en France (cf. thèse de M. Debien, Le Club Massiac).

63. Moreau de Saint-Méry, op. cit. 1: 17. 
(à M. de Navailles-Bonnas - 14 janvier 1790) $)^{64}$

Vous êtes parti tranquille sur le sort de nos colonies. Tout y est-il calme, la liberté, ce grand mot et qu'on fait sonner si haut, s'est-il fait entendre des nègres? Avez-vous éprouvé quelque mouvement dans vos habitations?

En réalité, en janvier 1790, malgré la grande peur d'un soulèvement général, à l'occasion des fêtes de Noël et du Jour de l'an, il n'y a pas de menace directe de mouvement dans les ateliers. Mais ce sont les mulâtres qui remuent çà et là, les gens de couleur libres qui réclament l'égalité des droits civiques, avec les blancs et à l'Arcahaye et à Montrouy où peu de propriétaires résident, ils sont nombreux et quelques-uns très riches. Puis la question des élections à l'assemblée coloniale qui doit se réunir à Saint-Marc agite et divise les colons. La première lettre des Terrien va tranquilliser Navailles. Tout est calme sur la plantation et ce sera le thème incessant des autres lettres qui, mettant face à face, la paix de l'habitation et l'agitation extérieure, porteront le maître lointain à attribuer ce tour de force à l'habileté des procureurs. Toute lettre d'administrateur est d'abord une apologie. Les colons ont réduit le plus qu'ils ont pu les répercussions des événements de France dans la colonie. La situation politique générale à Saint-Domingue est analysée par les Terrien: c'est le problème de l'esclavage et plus immédiatement celui des gens de couleur qui se pose après la Déclaration des Droits de l'Homme. Les colons ont pris des mesures de police. Les mulâtres se groupent et s'arment.

(à M. de Navailles-Bonnas - Saint-Mare, 6 mai 1790) ${ }^{65}$

L'ennemi le plus redoutable, écrivent les Terrien, que nous puissions y avoir, le nègre, démontre par continuation, la plus heureuse tranquillité. Nous regarderons toujours un soulèvement général comme impossible et des soulèvements partiels seraient bientôt étouffés.

Vous nous parlez des mulâtres comme nous en parlerions nous-mêmes si nous n'avions pas vếcu en Amérique; ils tiennent de nous leur état et leur liberté que nous ne leur avons donnée qu'à des conditions qui doivent toujours sub-

64. Correspondance Navailles-Bonnas.

65. Correspondance Navailles-Bonnas. 
sister. La Déclaration des Droits de l'homme a-t-elle été assez puissante pour donner aux juifs comme à tous les Français un état-civil dans toute sa plénitude? Le décret prononcé à cet égard sera-t-il bien accueilli? Cependant, cette nation depuis trop longtemps malheureuse n'a contre elle que l'opinion et une malédiction prophétique aussi incertaine que ridicule. La couleur du mulâtre, sa naissance d'un esclave afriquain, sont des signes de réprobation bien autrement marqués. Au surplus, comme la loi du plus fort sera éternellement la loi de tous les temps et de tous les pays comme c'est elle qui a opéré en France la Révolution, de même icy, à l'aide de cette loi, le blanc saura toujours maintenir la ligne de démarcation tracée entre lui et le mulâtre. Elle est indispensable politiquement considérée et même elle ne blessera pas l'équité naturelle autant que le préjugé sera maintenu à l'abri de loix sages et humaines. La leçon nécessaire que nous avons donnée aux mulâtres de notre dépendance a rétabli la plus parfaite tranquillité et elle durera.

C'est l'optimisme de la force victorieuse. Les libres des quartiers de l'Artibonite et des Verettes avaient en effet refusé le serment civique qu'on exigeait des électeurs, alors qu'eux-mêmes n'étaient pas électeurs. Illégalement l'assemblée électorale de l'ouest exigeait même que les libres devaient à la formule générale ajouter la promesse de respect aux blancs ${ }^{66}$.

Les libres des Verettes et de l'Artibonite prirent les armes. Ils étaient riches et nombreux. Ils se réunirent à l'habitation Pleasec, à la Petite-Rivière et appelèrent à l'appui de leur cause tous leurs frères de la colonie. (janvier 1790). Le soulèvement d'ensemble qu'ils avaient escompté ne se produisit pas. L'alarme n'en fut pas moins générale parmi les blancs. Le Cap et d'autres villes envoyèrent à Saint-Marc l'élite de leur garde nationale. A l'approche de ces détachements, l'attroupement se dispersa. Tout finit par quelques arrestations. Peut-être la modération de cette répression s'explique-telle par les craintes des grands planteurs qui voyaient monter la haine des petits blancs et se réservaient l'alliance des riches planteurs de couleur nombreux dans ce quartier des mornes. Mais l'amnistie promise se fit attendre longtemps.

66. D'après le P. Cabon, Histoire d'Haïti - Port-au-Prince (4 vol. Port-auPrince), III: 35 . 
Le point de vue des Terrien au sujet des mulâtres est plus brutal; il représente une partie de l'opinion des colons blancs. C'est surtout par leur nombre que les mulâtres alarmaient les blancs. Seule l'insurrection pouvait leur permettre d'obtenir satisfaction. C'est pourquoi les Terrien pensent comme beaucoup de colons blancs qu'en cas de rebellion des gens de couleur, il faut utiliser la force. Ils n'ont confiance qu'en elle pour maintenir une ligne de démarcation entre les couleurs. Sauf pour quelques sages, il n'est donc pas question d'une égalité politique entre les mulâtres libres et les blancs.

La situation va s'aggraver rapidement durant cette année 1790. Dans le Nord, au Cap, à l'ouest, au Port-au-Prince, des troubles sérieux apparaissent.

(même lettre).

...au Cap, il y a eu des complots réels, un chef a été découvert, la procédure instruite dans les formes, pendu de même. Un second chef est actuellement détenu et poursuivi aux mêmes fins.

Des événements plus malheureux ont eu lieu au Portau-Prince, quartier du Fond-Parision. Une dispute d'intérêt s'est élevée entre un mulâtre et un blanc imprudent et indiscret comme il y en a trop. Les plaintes ont attiré un détachement des troupes patriotiques, trois blancs ont été tués par les mulâtres soulevés, et cachés dans les bois; encore un quatrième blanc massacré depuis, un détachement de troupe est allé sur les lieux; point de mulâtres, ils se sont réfugiés dans des endroits inaccessibles. On brûle et on dévaste les propriêtés et, d'une inconséquence, d'une querelle particulière, on a fait une affaire générale. Elle ne peut avoir de suites funestes pour la colonie; mais ce sont des évênements d'autant plus malheureux qu'avec un peu de prudence, on les aurait évitês.

Peut-être. Mais l'atmosphère est trop tendue; trop chargée de haines ${ }^{67}$; la guerre de couleur couve depuis trop longtemps pour

67. et 68. Le précis historique des Annales de la Colonie française de SaintDomingue relate cet épisode de la révolution dans l'île d'une façon à peu près identique, avec cependant des détails plus circonstanciés sur l'affaire. La lettre des procureurs et le précis historique nous permettent de faire la chronique du quartier en avril 1790. A l'origine de l'affaire est l'économe gérant d'une habitation du FondParisien au quartier de la Croix des Bouquets. Le 26 avril 1790, il porte "à l'assemblée provinciale de l'Ouest, des plaintes bien ou mal fondées sur quelques difficultés 
que tout incident ne tourne pas en bagarres et en meurtres. On le voit par les détails plus précis de l'affaire du Fond-Parisien au quartier de la Croix des Bouquets ${ }^{68}$.

Massacres de blancs, soulèvement de mulâtres, dévastation des propriétés, telle est la situation au Montrouy, en mai 1790. Tout n'est donc pas aussi calme que voudraient le faire croire les frères Terrien et le quartier du Fond-Parisien n'est pas à une très grande distance du Montrouy. Mais c'est toujours la même attitude d'optimisme pour que le maître leur laisse le plus d'indépendance possible:

...N'ayez point d'inquiétude au sujet des noirs, encore moins pour les mulâtres. Et ce qui est la plus grande conclusion à leurs yeux, le résumé de toutes les causes de troubles: "Nous sommes nous-mêmes nos plus grands ennemis."

$\mathrm{Au}$ mois d'avril 1790 , s'est réunie l'assemblée coloniale de Saint-Domingue. Cette assemblée, comme d'ailleurs les autres assemblées coloniales, était un des grands désirs des planteurs et à leurs yeux, un moyen très efficace de lutter contre l'esprit métropo-

survenant entre lui et des hommes de couleur, propriétaires d'une sucrerie voisine, de celle qu'il régissait. Le comité en réfère d'abord à l'officier major commandant alors dans la ville (de Port-au-Prince) qui répondit qu'il allait mander ses honmes pour les entendre et les punir s'ils étaient coupables. Cette réponse était conforme aux lois et à ce qui s'était pratiqué de tout temps en pareil cas. Le comité le prend pour un déni de justice et dépêche sans information sous la conduite de l'imprimeur Nozard, 25 hommes de bonne volonté pris dans la galerie qui l'entourait pour aller les arrêter et les amener au Port-au-Prince. Prévenus de la violence que l'on se préparait à exercer contre eux, ils s'entourent de leur famille et se tiennent cachés et sur la défensive. Cette horde de bandits - quel autre nom leur donner? - ne trouvant pas ces hommes chez eux, fouillent leur maison et emmènent leurs enfants qu'ils y avaient laissés en leur criant qu'ils ne les leur rendraient que lorsqu'ils viendraient eux-mêmes les réclamer au comité du Port-au-Prince. A ce mots, un coup de fusil atteint l'un de cette horde. Il n'était que blessé. Tandis que les uns le relèvent et vont le déposer sur l'habitation voisine, les autres pressent leur retour au Portau-Prince, avec leur proie. Une réflexion un peu tardive pour leur camarade les décide à laisser les enfants sur la première habitation qu'ils rencontrent. Mais la rage n'en égare pas moins les pères. Lorsqu'ils ne les retrouvent plus chez eux, ils courent sur l'habitation voisine, source des maux qui les accablaient en ce moment; ils y immolent à leur vengeance le blessé et deux malheureux ouvriers blancs, attachés à cette habitation aux soins desquels il avait été laissé. Une partie d'entre eux se portent dans la montagne voisine, $y$ assassinent aussi un quatrieme blanc qu'ils soupçonnaient d'intelligence avec l'économe gérant de cette habitation. Le comité requiert alors la force armée pour avoir justice de ces meurtres. Le commandant expédie aussitôt contre cette famille un détachement de 50 hommes du régiment de Portau-Prince auquel se joignit une foule de volontaires de la ville et de la plaine. Cette expédition n'aboutit qu'à l'incendie et à la dévastation totale des biens appartenant à ces hommes de couleur. 
litain de l'administration royale et l'arbitraire des commandants militaires. Par ces assemblées se dessine un fort courant en faveur d'une autonomie coloniale, politique et économique, ou tout au moins du droit de la colonie à statuer seule sur son régime intérieur.

C'est ainsi que l'assemblée de Saint-Marc a des vues d'indépendance et la correspondance de Villevaleix, l'un des gérants des habitations Bréda ${ }^{69}$ confirme aussi ce point de vue.

De toute façon, les décrets et instructions du 8 mars et du 28 mars 1790, concernant les futures assemblées coloniales et les droits politiques des gens de couleur sont très diversement reçus par la population de l'íle. Ces décrets sont accueillis très froidement par l'assemblée. En effet, le décret du 8 mars, interprété par l'instruction du 28 mars a garanti à la colonie une certaine autonomie législative, mais a laissé sur les droits des gens de couleur libres, un voile qu'on a cru de grande politique. Et les interprétations vont leur train à la colonie: les uns se disent satisfaits d'un texte qui repousse les prétentions des mulâtres, puisqu'il ne leur accorde pas expressément le droit de vote et les Terrien sont de ceux-là. Les autres, en majorité à l'assemblée, voudraient un texte arrêtant net les ambitions des libres.

Aussi, très suggestive est l'opinion des frères Terrien sur l'assemblée générale de la partie française de Saint-Domingue. Ce point de vue des procureurs révèle l'état d'esprit très caractéristique a une partie de la population à l'égard de l'assemblée et de la mère patrie.

\section{(même lettre)}

...L'assemblée générale de la partie française de SaintDomingue, poursuivent les Terrien, a fait son ouverture solennelle le 14 avril.

Les esprits impartiaux, sages, modérés, n'y forment pas la majorité ; aussi beaucoup de travaux et rien de fait encore vraiment pour le bien public. Sans s'être donnée encore une attribution, un caractère positif, elle opère d'une manière

69. Villevaleix écrit le 30 juin 1790: “...L'assemblée provinciale du Nord a rendu un décret le 17 mai contre celui du 10 de l'assemblée de Saint-Marc qui culbute les vues d'indépendance de cette assemblée permanente dont le moindre mal serait de ruiner la colonie... La majorité des paroisses du Sud et de l'Ouest se sont déclarées pour l'assemblée de Saint-Marc... M. de Peinier n'a pour lui que la province du Nord, mais qui tiendra bon. Reste à savoir si ce qui résultera de cette désunion ne sera pas mauvais pour la colonie." 
toute puissante, elle a frappé quelques coups d'autorité déplacés pour détruire l'ancienne autorité.

On se plaignait amèrement des mauvaises dispositions de l'assemblée nationale contre la colonie, arrive son décret du 8 mars, vrai modèle de sagesse. Eh! bien! non seulement beaucoup l'accueillent froidement, mais encore telle est la bizarrerie de l'esprit humain, qu'on se dispose à l'éplucher de la manière la plus incroyable. Il n'y a cependant qu'une seule chose à redire, la sanction provisoire des décrets de l'assemblée coloniale qui ne peut être accordée raisonnablement à un gouverneur venu pour administrer une colonie sans la connaître, qui la quitte au bout de trois ans, alors qu'il commence à la connaître.

Mais la question la plus délicate est celle du commerce.

...L'assemblée deviendra chaude surtout quand on traitera les rapports commerciaux de la colonie avec la métropole.

Terrien considère donc le décret du 8 mars comme un vrai modèle de sagesse. C'est que ce décret du 8 mars fut pris sous l'impulsion de Barnave à la Constituante. Et Barnave était en relations avec les propriétaires absentéistes et les nobles du club Massiac. Les Terrien ne savent rien sans doute du travail occulte du Club des colons et de son alliance avec le Comité des Colonies de la Constituante, mais ils savent sans aucun doute, par Navailles-Bonnas que le décret a été préparé après consultation des colons.

Le décret était conforme au désir des colons; ils vont pouvoir élaborer leur propre constitution, réformer le système protecteur; ces deux dernières mesures devant être soumises à l'approbation de l'assemblée nationale.

Pas un mot direct dans ce décret sur les esclaves et les mulâtres. Mais les propriétés des colons sont placées sous la sauvegarde particulière de la nation. C'est une assurance. Naturellement, les Terrien prévoient de difficiles discussions, quand sera débattue la question du régime de l'Exclusif. Ils sont commerçants et ne l'oublient pas sous les mots qui veulent être prudents.

(même lettre).

...Deux principales classes d'hommes partagent ce pays:

l'habitant et le commerçant. Auparavant d'être habitant ou commerçant, on devrait être homme juste et impartial; l'habitant ne l'est point; en génêral, au lieu de penser à corriger 
les abus seulement et ménéger les rapports justement établis, on paraît vouloir trop couper dans le vif, prescrire au commerce quelques lois injustes.

Et ici, bien qu'avec précaution, les Terrien prennent position en faveur des commerçants. Ne sont-ils pas eux-mêmes commerçants? La France est-elle disposée à respecter les droits du Commerce? C'est la réapparition de l'esprit d'autonomie des "Américains", et ceux-ci n'ont pas reculé devant la révolte déjà en 1722 et en $1763-1769$.

Le temps, les réflextions, ajoutent les procureurs, surtout l'empire de la raison, finiront par se faire entendre peut-être; tant pis si on est sourd. La métropole est forte créancière de Saint-Domingue. Saint-Domingue est riche et très faible... La métropole est puissante et, à défaut de la raison, la force la mettra toujours au-dessus des lois injustes qu'on voudrait lui imposer; mais de là des maux qu'on éviterait avec des sentiments de justice et d'impartialité.

Quelques paroles rassurantes à la fin de la lettre:

Telle est, Monsieur, l'état actuel des choses à SaintDomingue; n'en concluez rien de fâcheux pour vos intérêts. En dernier résultat, les travaux de l'assemblée tourneront infailliblement à l'avantage de l'habitant et vous êtes habitant de Saint-Domingue par vos propriétés territoriales...

Un post-scriptum, daté du 6 mai 1790, est plus pessimiste:

L'assemblée a arrêté qu'il serait écrit une lettre à l'assemblée Nationale et une au roi su sujet du décret du $8 \mathrm{mars}^{70}$. Nous sommes persuadés que ce seront plutôt de belle phrases vagues que des expressions de reconnaissance; on croit n'en pas devoir; il est question de considérer Saint-Domingue comme province fédérale de France et autres sistèmes tendant trop à altérer l'union fraternelle.

La métropole sera défendue, mais la majorité parâ̂t devoir atre pour traiter la mère patrie avec injustice et ingratitude". Elle aura toujours icy de bons enfants; de là des partis op-

70. La colonie connaissait depuis la fin d'avril le texte de ce décret, mais l'assemblée générale ne l'avait pas encore reçu officiellement. Par sa lettre, l'assemblée générale voulait réclamer contre ce décret, pour le faire modifier avant son arrivée officielle. C'était pure fiction. Elle n'avouera l'avoir reçu que le ler juin. Elle avait eu tout le temps de se remuer pour le faire rapporter. 
posés, des dissensions qu'on peut redouter sans néanmoins s'en affliger d'avance.

Nous ne sommes qu'au début d'une longue lutte que la guerre de couleur tournera chez beaucoup de colons en volonté décidée d'indépendance. Dès 1790 , une majorité se forme, prête à détendre les liens qui unissent la colonie à la métropole.

M. G. Debien a parfaitement analysé cet état d'esprit lorsqu'il écrit: "Les correspondances venues de Paris, des alentours de l'Assemblée Nationale et du Club Massiac, énervent les propriétaires, dressent les uns contre les autres, colons résidant à SaintDomingue et propriétaires coloniaux touchant en France les revenus de leurs lointaines plantations"'71. - Au début, la plus grande partie de ceux qui prirent le parti de la Révolution à Saint-Domingue, le firent avec l'arrière-pensée de rendre l'île entière libre de ses destinées.

La lettre du 15 juillet 1790, merveilleuse de franchise politique et relatant les principaux événements survenus à Saint-Domingue, du 15 juillet au 20 juillet, mérite d'être citée intégralement.

C'est un véritable journal permettant de voir se dérouler à SaintDomingue, au jour le jour, les faits essentiels. Dans cette lettre, les Terrien confirment la position qu'ils ont prise précédemment. Le 28 mai, l'assemblée coloniale a posé dans un décret solennel, voté à l'unanimité, les bases constitutionnelles du régime qu'elle entend se donner. C'est la thèse des autonomistes qui triomphe. SaintDomingue fera pratiquement seule des lois intérieures. C'est la meilleure manière qu'elle ait trouvé pour résoudre ou plutôt pour dominer la question des libres et celle de l'esclavage. C'est la thèse des colons blanes, la thèse de la semi-autonomie.

La situation s'aggrave dans l'île. Dans le Nord, c'est le soulèvement du Cap, siège de l'assemblée provinciale du Nord, qui attaque l'assemblée coloniale. Au Cap, l'assemblée provinciale du Nord où les négociants font nombre, vise à son indépendance particulière et attaque l'assemblée coloniale qu'elle accuse de rompre avec la métropole. La correspondance de Villevaleix ${ }^{72}$, à cette même époque, confirme cet état de choses.

71. Souligné dans le texte.

72. G. Debien, op. cit. 
C'est l'anarchie complète, d'autant plus qu'il y a conflit entre la municipalité et l'assemblée provinciale du Cap ${ }^{73}$.

En opposition avec Villevaleix, les Terrien soutiennent l'assemblee coloniale ${ }^{74}$.

Cependant l'assemblée de Saint-Marc est confirmée par le gouverneur général le 13 juillet à une majorité apparente de 90 voix contre 48 sur 165 suffrages que donnait la totalité des paroisses de la colonie en comptant 1 député pour cent citoyens actifs (40 paroisses sur 51 avaient envoyé le résultat des votes). Beaucoup des paroisses confirmaient l'assemblée à la charge de se conformer aux décrets des 8 et 28 mars.

C'est au moment où ces événements se déroulent dans l'île que les procureurs vont faire un long récit à leur maître en France.

(à M. de Navailles-Bonnas, Saint-Marc, 15 juillet 1790. $)^{75}$

...Il était presque impossible que la première assemblée générale d'un pays encore neuf, qui n'avait jamais connu que des assemblées de paroisses mal ordonnées, n'eût pas un début défavorable; celui de notre assemblée coloniale l'a été; en effet, il a été maladroit, peut-être et cela par la faute de quelques membres véhéments, inconsidérés, dont les propos ou les actions étaient irréfléchis; le public en a tiré des conséquences trop forcées, injustes; l'assemblée est redevenue

73. Villevaleix écrit au 31 juillet 1790 de la Plaine du nord: "...on a fait de suite partir une députation auprès du général pour l'engager à soutenir notre armée par une seconde du Port-au-Prince, pour en imposer davantage à celle de Saint-Marc, aux ordres de $\mathbf{M}$. de Cadusch. Malgré l'insurrection du Port-au-Prince, malgré le massacre de $M$. de Codère et de son fils, ...cette assemblée a beaucoup de partisans. On craint beaucoup une réunion de toutes les paroisses antinationales pour secourir l'armée de Saint-Marc. Alors le mal serait à son comble et la colonie au néant. Voilà notre point de vue.

74. Garran - Coulon, Rapport sur les troubles de Saint-Domingue, 1199. Les quatre commissaires de l'assemblée coloniale. Jouette père, député de l'Arcahaye. Barillon, député du Cap, Valentin de Cullion, fils, député du Petit-Goave, Duclaux, député du Cap, au lieu de se présenter à l'assemblée du Nord, avaient commencé par descendre chez un officier municipal, Chauvain, et par tenter de déclencher un nouvement des districts du Cap contre l'Assemblée du nord, Lors de leur réception à l'assemblée, ils avaient cherché à ameuter les galeries. Au bout de quelques jours, l'assemblée du Nord enjoignit le 15 juin par un brusque arrêté à Jouette et à Valentina de Cullion de quitter le Cap le jour même et la province du Nord dans les 48 heures, à Duclaux et à Barillon de rester au Cap. C'était couper les points avec Saint-Marc.

75. Correspondance Navailles-Bonnas. 
beaucoup plus sage, mais la prévention du public, loin de diminuer, a augmenté; de là, des rumeurs, des partis, des cabales pour et contre l'assemblée. Est arrivé le décret du 8 mars qui, au lieu d'apporter la paix, dans l'esprit de laquelle il était fait pour la colonie, y a malheureusement augmenté le désordre et il en a été de même des instructions décrétées le 28 mars, subséquentes au décret du 8. Les adversaires de l'assemblée se sont appuyés de ces deux pièces, ont prétendu qu'elle devait s'y conformer aveuglément; celle-ci au contraire, y a beaucoup dérogé, en posant des bases constitutionnelles dans son décret du 28 mai.

Elle ne veut point de sanction provisoire du gouverneur, parce qu'elle dit que la sanction ne peut être dévolue à celui qui n'est pas inviolable; alle veut faire seule absolument ses lois pour son régime intérieur; elle veut envoyer ses décrets à l'assemblée nationale et à la sanction du roi et provisoirement, elle veut faire exécuter toutes ses lois pour l'intérieur; le général ${ }^{76}$ s'y refuse, disant que la nation et le roj lui ont littéralement prescrit ses devoirs dans les décrets des 8 et 28 mars qui les rendent personnellement responsables de tout ce qu'il pourrait y faire de contraire ${ }^{77}$.

Comme conséquence de cette situation: "Voilà donc le pouvoir législatif, arrêté, empêché, par le pouvoir exécutif, continuent les Terrien, source naturelle d'anarchie, difficile à terminer qu'après que la nation aura prononcé. La ville du Cap surtout, forte par sa population, sa richesse, donnant elle seule 24 députés sur 212 , la ville du Cap est soulevée, déchaînée contre l'Assemblée Coloniale. Elle est le siège de l'assemblée provinciale du Nord; celle-cy au lieu d'être forte seulement de bonnes raisons, au lieu de s'appuyer froidement

\section{Le Gouverneur Général.}

77. Le précis historique n'est pas tout à fait d'accord à ce sujet avec l'auteur de la lettre. Il est question, en effet, non d'approbation de l'assemblée nationale et de sanction du roi, mais de leur acceptation. La nuance vaut la peine d'être signalée:

"Enfin, (son) fameux décret du 28 mai, est-il dit dans le précis, ne laisse plus de doute sur l'étendue de son système. Les décrets de l'Assemblée Nationale n'étaient pas encore parvenus dans la colonie, mais ils commençaient à y être connus et ce fut ce qui accéléra l'émission de ce décret, où elle tâcha d'établir les bases constitutionnelles de la colonie. Elle soumettrait ce décret non à l'approbation de l'Assemblée Nationale et à la sanction du roi, mais à leur acceptation et se considérant déjà comme n'ayant avec la France que des relations purement commerciales et d'alliance, elle prétendait donner une exécution pleine et entière à ce décret sans attendre ce qu'en pourrait penser l'Assemblée Nationale et le roi, dans la confiance où elle était que dans le cas où ils refuseraient de l'accepter, elle pourrait compter sur la bonne volonté de l'Angleterre qui ne demandait pas mieux que d'agrandir son commerce par l'addition d'une aussi belle colonie au nombre de ses alliés." 
des principes de l'Assemblée Nationale, attaque injurieusement la Coloniale; celle-cy lui envoie quatre commissaires ${ }^{78}$ pour s'entendre et se concilier; elle les proscrit, les menace de les appréhender au corps et de les envoyer à l'Assemblée Nationale, et à chaque arrêté, cette assemblée provinciale pèche par les plus grandes inconséquences aux principes mêmes dont elle veut s'appuyer.

Villevaleix, dans sa correspondance, relate également ces faits, notamment la forte opposition entre le Nord et Saint-Marc. Terrien continue:

Enfin, pour se conformer au décret du 28 mars, les paroisses se rassemblent de nouveau; sur 212 voix, il y en a 130, pour confirmer l'assemblee, purement et simplement, un petit nombre pour en former une autre et un assez grand nombre pour la maintenir en l'astreignant aux décrets de l'assemblée nationale des 8 et 28 mars.

Voilà done trois opinions bien opposées; cependant le vœu général est pour l'assemblée; elle n'est pas plus forte parce que le pouvoir exécutif s'oppose toujours avec effet et soutient les détracteurs de l'assemblée.

Mais déjà l'assemblée provinciale du Cap est aux prises avec la municipalité; encore quelques jours et cette assemblée provinciale sera anéantie par les citoyens ou les citoyens du Cap seront armés les uns contre les autres.

Villevaleix de la Plaine du Nord, signale le 31 juillet 1790 , les dangers de cette division, il voit le "mal à son comble et la colonie au néant."

L'Assemblée Nationale, poursuivent les procureurs, a accordé les facultés de mettre à exécution la partie du décret des municipalités qui peut s'adapter à la colonie, sauf à y faire les modifications qui nous sont nécessaires et qu'on devra sanctionner auparavant l'exécution; et point du tout, l'assemblée a coupé, élégué, ajouté, modifié; ces changements sont bons, sans doute, mais ils changent tout icy dans la judicature; il leur faut une sanction et on veut néanmoins établir ces municipalites sans aucune sanction; mais le gouverneur s'y oppose; néanmoins, la plus grande partie des paroisses les établiront; les commandants seront forcés dans leur vo-

78. Les quatre commissaires de l'assemblée coloniale sont Jouette père, député de l'Arcahaye, Barillon, député du Cap, Valentin de Cullion fils, député du PetitGoave, Duclaux, député du Cap. 
lonté contraire; les cours de justice s'opposeront sourdement, et de là, beaucoup de schisme.

La vérité est que l'assemblée générale de la colonie s'est un peu aveuglée sur ses pouvoirs, elle a voulu aller trop vite; au peuple de Saint-Domingue seul appartient de faire ses lois, lui seul connaît celles qu'il lui faut; il lui faut une constitution particulière, celle de France l'anéantirait.

Terrien est dans ce passage d'une franchise étonnante; il est vrai qu'il ajoute:

Mais ce peuple n'est qu'une portion de la nation; ses plans, sa constitution rédigés par lui, doivent être décrétés par la nation, sanctionnés par le roi, ensuite exécutés icy, et on ne devrait exécuter provisoirement que ce qui est de la plus urgente nécessité; mais l'assemblée coloniale ne croit pas devoir être aussi bornée.

Enfin, aujourd'hui 15 juillet, elle a décrété que le pouvoir législatif et le pouvoir exécutif devaient être réunis, 4 commissaires partent demain, vont trouver le général, pour l'inviter à venir auprès de l'assemblée ou pour que l'assemblée aille tenir ses séances auprès de lui. Le résultat de cette démarche sera d'une grande importance; mais tout dépendra de la manière dont l'Assemblée Nationale considérera les bases constitutionnelles de l'assemblée coloniale, décrétées le 28 mai, expédiées le ler juin. Se tiendra-t-elle rigoureusement à ses principes? Nous ne le croyons pas; nous pensons que dans le moment d'une révolution, elle jettera un voile politique sur les empiétements de l'assemblée coloniale. Dans tous les cas, voicy une vérité incontestable: tous les habitants de Saint-Domingue, sans exception, sont bons Français, fiers de l'être, à jamais fidèles à la nation française, et jamais la plus légère atteinte ne sera portée à ses sentiments qui sont ceux de l'assemblée coloniale, comme des derniers citoyens...

L'admirable décret de l'Assemblée Nationale du 22 mai sur le droit de la guerre et de la paix, a fait une bienheureuse diversion à l'état fâcheux de nos affaires publiques; il a produit la sensation la plus vive, la plus délicieuse et générale. Nous croyons avoir évité la guerre, pour le moment, et nous pensons que quand nous l'aurons, la colonie sera bien autrement protégée que cy-devant. On ne sait qu'admirer le plus dans ce décret, de sa magnanimité, de sa philosophie, de sa politique ou de sa sagesse. Tous les colons en sont ravis, ils pensent avec raison que leurs propriétés ont acquis une grande augmentation de valeur par les suites et les effets de ce sublime decret. 
- 19 juillet - Les choses viennent de changer de face tout à coup, au Port-au-Prince, siège de l'administration, du pouvoir judiciaire et il en résulte beaucoup de défaveur pour l'assemblée coloniale qui était fortement appuyée par le comité et par ce qu'on nomme la multitude. Tout-à-coup s'est élevée une compagnie de 600 volontaires, composée de l'élite des citoyens de la ville qui, à son tour, en impose absolument à la multitude et au comité; elle a prononcé le serment suivant:

"Nous, Français, de la paroisse de Port-au-Prince, icy rassemblés en corps de volontaires, jurons et promettons par les loix de l'honneur, de nous soutenir et secourir dans toutes les occasions et de nous réunir d'esprit, de cœur et d'action, à tous les bons citoyens qui, n'abjurant point leur mère patrie, adoptent comme loi sacrée et fondamentale les décrets de l'Assemblée Nationale, en date des 8 et 28 mars et les instructions adoptées par ladite assemblée; promettons en outre, de protéger et défendre l'Assemblée Coloniale de Saint-Domingue" qui sera reconnue par le vœu général de la colonie en tant qu'elle ne s'écartera jamais, sous aucun prétexte, des décrets de l'assemblée Nationale, cy-dessus rappelés - Au Port-auPrince, le 12 juillet 1790 .

A ces faits, se joignent des bruits vrais ou faux, suivant lesquels le Président du Comité de Port-au-Prince ${ }^{79}$ et quelques membres auraient cherché à gagner des soldats du régiment de Port-au-Prince pour assassiner le général ${ }^{80}$, leur colonel ${ }^{81}$ et autres têtes majeures et opposées à l'assemblée coloniale; deux soldats sont aux fers, on instruit leur procès, le président du Comité est dénoncé à la partie publique; mais il reste dans la ville sans témoigner d'inquiétude; beaucoup de rumeur sur l'annonce des 4 commissaires de l'assemblée coloniale; de tout cela résultera une augmentation d'anarchie, mais dans ce moment, dans l'état actuel des choses, le résultat paraît être que le parti le plus fort dans la colonie est celui qui veut maintenir purement et simplement les décrets de l'Assemblée Nationale et seulement ceux des décrets de l'assemblée coloniale que la Nationale aura décrétés et le roi sanctionnés. Les volontaires du Cap, du Port-au-Prince et de Saint Marc sont absolument unis pour maintenir cette opinion; ils sont forts, déterminés, nombreux et se recrutent tous les jours.

79. Caradeux aîné, grand planteur, célèbre par sa dureté à l'égard de ses esclaves.

80. Le comte de Peinier. Peinier.

81. Le Chevalier du Mauduit du Flessis, parent d'ailleurs du gouverneur 
Il semble donc, d'après les Terrien qu'à la date du 19 juillet 1790, une forte majorité se constitue à Saint-Domingue, soutenant l'Assemblée Coloniale et prête à se soumettre aux décrets de l'Assemblee Nationale et du Roi. Villevalei $x^{82}$, dans sa correspondance, n'est pas du tout de cet avis et note au contraire, dans sa lettre du 31 juillet 1790 une forte opposition à l'Assemblée Coloniale et au mouvement dirigé de Saint-Marc.

Les Terrien semblent d'ailleurs pêcher par optimisme, car ils 6 crivent le 20 juillet:

-20 juillet - Les quatre commissaires envoyés vers le général, reviennent demain, sans aucun succès dans leur mission, le général, M. de Peinier, bon eitoyen, mais faible, peu de génie, mal conseillé, n'a voulu traiter l'affaire publique avec eux que par correspondance; il leur a d'ailleurs objecté que les ordonnances du roi lui prescrivaient sa résidence au Port-au-Prince, qu'il ne pouvait done se rendre à Saint-Marc, qu'il sentait le besoin du rapprochement du pouvoir exécutif du législatif, mais qu'il ne reconnaissait ce dernier que dans l'Assemblée Nationale.

D'un autre côté, l'Assemblée provinciale du Nord, a pris absolument le dessus, a détruit sa municipalité qui était portée pour l'Assemblée Coloniale, de manière que voilà celle-cy fortement empêchée, arrêtée et cela durera jusque vers le mois d'octobre, époque de la réception des nouvelles de l'Assemblée Nationale relativement aux bases constitutionnelles de celle d'icy. La colonie se trouve done dans la plus fâcheuse position et de tous les états, le commerçant est celui qui en souffrira le plus dans ses intérêts, dans le long intervalle de l'anarchie au bon ordre.

-20 juillet 1790 - A trois assemblées constitutives de notre paroisse, nous avons travaillé à l'élection de nos officiers municipaux, lutté contre une cabale infernale qui voulait nous en donner d'indignes; heureusement, elle a eu le dessous et déjà nous avons un maire, un procureur syndic, et trois premiers officiers dont nous pouvons nous applaudir et nous glorifier. On peut espérer que la suite ira bien et au moins pourrons-nous prétendre au bon ordre dans notre ville.

Notre ami, M. Castillon ${ }^{83}$ pourra vous communiquer

82. Correspondance Villevaleix - Arch. Loire Inférieure F. 691.

83. "Notre ami M. Castillon" doit être Abraham Castillon Duperron, marié à Anne-Madel. Brunaud, associé à J.-B. Donatien Terrien et à Jacques Augustin Terrien dans l'exploitation d'une caféière à la Rivière de Voldrogue à Jérémie, estimée 366.000 livres (Etat des indemnités, 1832). 
divers imprimés qui achèveront de vous mettre parfaitement au cours de la différence des opinions qui divisent maintenant la colonie de Saint-Domingue.

Cette longue lettre apporte à Navailles-Bonnas un écho précis des difficultés politiques dans lesquelles se débat la colonie. Elle n'est pas pour calmer les appréhensions du propriétaire résidant en France. Les Terrien continuent à soutenir le parti des colons blancs et, avec ce parti, le parti de la demi-autonomie. Les luttes politiques, qui cachent, la plupart du temps, des divergences d'intérêt, sont ardentes dans l'île. Le témoignage des procureurs, devant la nouvelle situation créee par la Révolution, est précieux. C'est une contribution à l'histoire politique de l'île dans cette année cruciale. Mais tout cela n'est guère encourageant pour le propriétaire, se demandant avec anxiété dans quel état vont se trouver ses biens.

Une nouvelle missive, datée du 8 septembre 1790 , va, tout en rassurant Navailles, sur sa caféière, lui apporter quelques renseignements nouveaux.

Depuis le mois de juillet, en effet, la situation politique a évolué à Saint-Domingue. L'assemblée de Saint-Marc avait adopté l'idée d'une véritable autonomie coloniale, ou du moins, elle voulait une colonie statuant seule sur son régime intérieur. La présence de députés de Saint-Domingue à l'assemblée nationale devenait dangereuse. Et il semble bien que sur ce point, l'assemblée de Saint-Marc pensait comme le Club Massiac. Aussi, lorsqu'elle avait connu les décrets des 8 et 28 mars 1790, "concernant les futures assemblées coloniales et les droits politiques des gens de couleur", (souligné dans le texte) avait-elle ordonné aux élus de Saint-Domingue, par décret du 28 mai 1790, de "suspendre toute fonction à l'assemblée Nationale, en qualité de députéte."

Les grands blancs étaient bien partisans de réformes, mais dans la mesure où ces réformes augmenteraient leur propre autorité.

D'autre part l'assemblée de Saint-Marc se prétendant menacée par le gouverneur Peinier et la force armée avait soulevé l'équipage

84. Souligné dans le texte. Correspondance Navailles-Bonnas. 
du vaisseau "le Léopard", outre son commandant, M. de la Galissonnière et s'était embarquée pour la France, les 6,7 et 8 aout. ${ }^{85}$

Les frères Terrien annoncent ainsi la nouvelle à leur maître:

(a M. de Navailles-Bonnas, -8 septembre 1790$)^{86}$. ...Quel aura été votre étonnement, Monsieur, en apprenant l'arrivée en France, sur le vaisseau de guerre "le Léopard", de l'assemblée coloniale proserite par la moitié de la colonie, plainte et admirée par l'autre moitié.

La paix et la tranquillité sont parfaites en ce moment; nous ne parlons pas des divisions particulières, inséparables de la diversité d'opinions; actuellement, les détracteurs de l'assemblée coloniale sont aussi fanatiques que l'étaient ses partisans, il y a deux mois... La politique n'est pas indifférente au commerce. ...il serait aussi nuisible à la métropole qu'à la colonie que l'assemblée revînt triomphante ou humiliée. Blâmer, pardonner, effacer, surtout réconcilier, voilà tout ce que l'assemblée nationale a à faire pour opérer le bien général.

Quelle sagesse politique dans cette conclusion de la part des Terrien! Surtout réconcilier, soulignent-ils dans le texte, mais malheureusement les passions politiques sont trop déchaînées en France et dans la Colonie, les interêts trop divergents pour arriver à ce résultat.

En 1791, nous n'avons pas de lettres Terrien relatives à la situation politique dans l'île et le départ du "Léopard", signalé dans la lettre du 8 septembre 1790, est la dernière nouvelle qu'ils nous donnent de la vie politique à Saint-Domingue, au début de la Révolution.

85. Correspondance Villevaleix. Villevaleix dans ses lettres parle abondamment des brigands du "Léopard". Dans la Plaine du Nord, on les a surnommés les "crochus". Les adversaires des "crochus" et de l'Assemblée de Saint-Marc sont les "Bossus".

Le 31 août 1790, Villevaleix écrit: "Les Bossus" ne peuvent se persuader que des brigands aient l'audace de se présenter aux Etats Généraux. Les Crochus prétendent qu'ils iront avec d'autant plus de raison qu'ils ont en France de très grands Crochus qui les protégeront, d'après le vœu desquels ils ont agi ici et que, par conséquent, il n'ont voulu que le bien de la colonie."

Le 30 septembre 1790 , la colère de Villevaleix contre l'assemblée n'a pas désarmé: “...L'arrivée de l'assemblée de Saint-Marc, en France, a dû faire une grande sensation aux amis du bien de cette colonie et si leurs forfaits ne sont punis sévèrement, la colonie, aux premières nouvelles, sera livrée à toutes les horreurs de la haine et de la vengeance..."

86. Correspondance Navailles-Bonnas. 
Ces longs récits des procureurs à leur maître en France présentent un réel intérêt.

L'année 1790 marque un tournant important dans l'histoire politique, économique et sociale de Saint-Domingue. Elle est capitale. Elle explique toute la suite de l'histoire de l'ṣle.

La vie politique de la colonie est transformée par la Révolution en France. Cette évolution est bien indiquée dans les lettres Terrien. Les désirs, mais aussi les fautes, les maladresses des colons, sont signalées; l'impatience des noirs et des mulâtres à s'émanciper également. Mais ces derniers ne sont pas encore aptes à exercer ces nouveaux droits. Les Terrien raisonnent comme les colons et les propriétaires blancs; leurs idées sont semblables à celles des membres du Club Massiac à Paris. De plus, leurs lettres sont l'indice des réactions du commerce devant la politique autonomiste de l'assemblée de Saint-Marc.

Les Terrien, dans leur île, ne réalisent pas pleinement l'importance des réformes édictées par la Constituante. Ils seront bientôt débordés et emportés par les événements. Ils veulent le maintien de l'ancien état de choses, le seul qui d'ailleurs à ce moment soit possible pour la prospérité de l'île, mais avec des libertés pour le commerce.

Enfin, les liens qui unissaient la colonie et la métropole allaient diminuant; les intérêts étaient trop divergents, les passions trop fortes. Saint-Domingue, en cette année 1790 , commençait à se détacher de la France.

Chapitre CINQUième

L'HABITATION SOUS L'OCCUPATION ANGLAISE (1793-1798)

Le 18 juillet 1793, an II de la République, le citoyen NAVAILLES-BONNAS reçoit du ministre de la Marine et des Colonies, un certificat de civisme ${ }^{87}$.

J'ai reçu le certificat de votre municipalité qui constate que vous résidez en France. Je vais prendre des mesures

87. Correspondance Navailles-Bonnas. 
promptes pour faire connaitre aux administrateurs des colonies que vous avez satisfait aux décrets relatifs aux propriétés des émigrés dans les colonies et que la République doit vous compter au nombre des bons citoyens qui lui sont restés attachés.

Dans une autre lettre du 1er jour des sans culottides, an II, le citoyen Navailles-Bonnas est classé parmi les bons "Français et loyaux républiquains". Il a donc donné des gages au nouveau régime pour conserver ses biens à Saint-Domingue. Navailles réside à ce moment à Bagnères-de-Bigorre et il apprend par cette même lettre la vente et la livraison de ses cafés par "La Marguerite". "Nous ne pourrons, dit cette lettre, vous en remettre le compte que lorsque nos contestations avec l'armateur, soit pour le frêt, soit pour le déficit, seront en règle." Malgré les temps troublés et l'insécurité des mers, les envois de café ont continué en 1793 de Saint-Domingue en France.

Pendant que Navailles donnait des gages au nouveau régime en France, à Saint-Domingue, un mulâtre, Lapointe devenait commandant de la garde nationale du quartier; c'était un modéré. Mais ni les blancs, ni Lapointe, ni les mulâtres ne reconnaissaient en fait l'autorité des commissaires civils envoyés par la Législative: Sonthonax ${ }^{88}$ et Polverel. Ils leur paraissent n'apporter que de nouveaux troubles sociaux.

88. Sonthonnax (1769-181s) - né le 17 mars 1763, à Oyonnax, d'une famille de commerçants. En 1789, Avocat au Parlement de Paris; en 1791, admis au nombre des avoués, près le Tribunal de Cassation. Membre très actif des Jacobins et des diverses sociétés patriotiques - collaborant aux Révolutions de Paris. Nommé commissaire civil pour Saint-Domingue, par le ministère girodin, il quitte Lorient le 22 juillet 1792. Il se présenta d'abord dans la colonie, en pacificateur et conciliateur pour faire accepter les mesures nouvelles à l'égard des mulâtres, mais en vain. Il s'appuya dès lors au cours des troubles sur les mulâtres et sur les noirs. C'est lui qui décréta l'affranchissement des esclaves, le 29 août 1793.

Avec le Général Laveaux, il combattit les Anglais et les Colons qui avaient pactisé avec eux. Obligé de s'embarquer pour la France après la reddition du Portau-Prince, il dut y répondre avec son collègue Polverel aux accusations dont il était l'objet. Les débats de la commission d'enquête se déroulèrent de février à octobre 1795 et Sonthonnax fut acquitté.

En janvier 1796, une nouvelle mission lui fut confiée à Saint-Domingue, avec Raimond, Le blanc et Giraud. Il fut en 1796 nommé député de Lille aux 500 où il siégea parmi les républicains avancés.

Consulté sans doute au moment de l'expédition de 1803, il devint suspect au gouvernement insulaire et impérial, fut éloigné de Paris et tenu en surveillance. Il mourut le 23 juillet 1813. 
La grande majorité des planteurs s'était ralliée comme l'indiquent d'ailleurs les Terrien, à l'union de Saint-Marc qui refusait d'obéir aux représentants de la Métropole. D'autre part, quand la déclaration de guerre avec l'Angleterre et l'Espagne fut connue à Saint-Domingue, en mars 1793, on n'approuva point à l'Arcahaye le ralliement de beaucoup d'officiers à l'Espagne. On n'applaudit pas davantage aux négociations que quelques colons d'aventure entamèrent à Londres, de février à juillet 1793 , pour assurer à SaintDomingue les bénéfices d'un traité d'occupation qui, sauf le mot, avait tout d'une simple capitulation. Des troupes anglaises avaient été débarquées à Saint-Marc, à quelques lieues de l'Arcahaye. Les Anglais occupèrent l'Arcahaye la veille de Noël (1793) ${ }^{89}$. Il n'y eut guère d'opposition de la part des colons blancs. Dans ce quartier épargné au moment des troubles civils et du soulèvement des esclaves, la plantation Navailles, comme nous aurons l'occasion de le voir, ne cesse de travailler, au ralenti, il est vrai. Car si la plantation continue tant bien que mal son travail, elle devait souffrir de tous les troubles que les biens des propriétaires eurent à subir aussi bien dans les quartiers restés aux commissaires civils que dans ceux passés aux Britanniques et Montrouy passa aux Anglais.

En janvier 1794, un mois après l'occupation anglaise, Terrien aîné quitte Saint-Domingue et passe à Savannah, en Géorgie. Son frère restera dans l'śle et le tiendra au courant des événements politiques survenus et de la situation de l'habitation. A son tour, Terrien aîné informera Navailles-Bonnas.

L'histoire des plantations, sous l'occupation anglaise est celle des biens vacants; les Anglais séquestrèrent toutes les plantations dont les propriétaires résidaient en France ou n'avaient pas donné signe de vie depuis un an.

Girodon qui avait succédé à Guchen, en qualité de gérant, est également passé en Amérique, à l'arrivée des Anglais. Il gérait l'habitation Navailles depuis trois ans, mais il n'a pu donner aucun détail sur la situation, la colonie étant dans l'anarchie au moment où il a pris ses fonctions. L'arrivée des Anglais a arrêté tout moyen

89. Montrouis se soumet aux Anglais en décembre 1793. (cf. Cel Chalmers, Remarks on the late warin San Domingo (London, 1803), 16-17). 
de correspondance. Sa vie étant menacée, il s'est vu forcé de passer comme bien d'autres à la Nouvelle-Angleterre.

Gérants et procureurs, dans le désordre de ces années troubles, ne sont guère d'accord sur les quantités de café récolté et leurs lettres feront état de leurs points de vue opposés.

(Girodon à Mme de Navailles-Bonnas, à Bordeaux, le 24 thermidor, an IV) ${ }^{20}$

...Je prends la liberté de vous écrire, Madame, au sujet d'une lettre qu'a écrit $M$. Terrien à votre mary et qu'il a bien voulu me communiquer, dans laquelle il lui dit que la récolte de 95 n'a produit que $\mathbf{4 1}$ milliers de café, mais que tous frais payés, il ne restait en votre faveur que deux mille livres. Mais il ne lui parle pas de celle de 94 qui est du même nombre et de celle de 95 qui est de près quatre-vingt; il est vray que sur cette dernière, je crois qu'il vous avait fait quelque envoi...

M. Terrien n'aurait pas dû s'en tenir à d'aussy faibles détails; ce serait pour vous une faible consolation qu'il vous eût produit des comptes, quy vous montrassent au clair l'emploi qu'il a fait de ses trois récoltes. C'est pourquoi, Madame, vous ne devez pas perdre espoir jusqu'à ce moment. Ce qu'il y a de bien certain, c'est que le sieur Terrien est à la Nouvelle Angleterre avec de grands moyens et que s'il veut, il peut faciloment vous faire quelque envoi; il le devrait d'autant plus qu'il doit actuellement avoir perçu une quatrième récolte, puisque l'Anglais permet aux procureurs de disposer des récoltes comme cy devant du moins jusqu'à son départ: Ce qui prouve que cela est encore, c'est que le sieur Terrien doit avoir des lettres très frâiches de son frère qui est sur les lieux, dans lesquelles il ne lui en parle nullement. Il se borne à dire, comme vous le verrez, par sa lettre, que votre habitation est en bon état...

Au départ de Girodon, il restait sur l'habitation encore cent vingt-cinq nègres, compte non tenu des recrues levées pour les régiments coloniaux. La plantation faisait assez peu de revenus, parce que les ateliers étaient continuellement détournés par les travaux de fortification. Girodon affirme dans la suite de sa lettre que l'habitation possède encore beaucoup de ressources.

90. Correspondance Navailles-Bonnas. 
Quelle est la position de Terrien vis-à-vis de ce que nous pouvons considérer comme un acte d'accusation de sa gestion ces dernières années?

Plusieurs lettres ont dû être échangées entre Terrien et Navailles-Bonnas, à ce sujet; elles ont malheureusement disparu des papiers de famille. Deux ans plus tard, en 1797, Terrien se défendra des accusations portées par Girodon au sujet de la récolte de 95.

En l'an IV, ce qui préoccupe Navailles-Bonnas, c'est la question des séquestres de ses biens. Il désire obtenir la levée du séquestre. C'est pourquoi de Navailles-Bonnas, devenu le citoyen Navailles, intervient auprès de son ami Turben, commis principal au Bureau des Colonies, pour cette question des séquestres indûment mis sur ses biens et la levée des revenus perçus pendant la durée de ces séquestres.

Le Bureau des Colonies est, en effet, chargé de dresser une liste des citoyens restés fidèles à la patrie. Turben fait en sorte que Navailles-Bonnas y figure, afin que la levée des séquestres puisse être faite "avec restitution des fruits". Cette liste est remise aux agents du Directoire exécutif, délégués à Saint-Domingue. Ces deux agents sont Raymond et Sonthonax. Turben insiste en faveur de son ami Navailles, par une note qui doit être remise à Raymond et à Sonthonax:

"Voila, citoyen, ce que j'ai cru devoir faire, écrit Turben, pour servir vos interêts et rendre justice à votre exactitude à vous conformer à la loi ${ }^{91}$."

Par la même occasion, Turben ajoute sur cette note de recommandation, François Maillat, neveu des Seguineau et Pelevin.

Notre remise aux citoyens Sonthonax et Raymond, agents particuliers du Directoire exécutif aux Isles-sous-le-Vent ${ }^{92}$.

De tous les propriétaires des colonies inscrits sur la liste qui vous est remise des citoyens restés fidèles à la patrie, il n'en est aucun dont les certificats n'ont été examinés avec la plus sévère attention. Ils ont tous dès lors un droit légal aux effets légaux de leur inscription qui sont la levée des sequestres, indûment mis sur leurs biens et la levée des revenus perçus pendant la levêe de ces sequestres.

91. Correspondance Navailles-Bonnas.

92. Papiers Navailles-Bonnas. 
Mais comme la suspension des communications a rendu le nombre des sequestres très considérables, les opérations d'une liquidation qui ne peut être que successive, rendra inévitablement lente pour plusieurs la justice qu'ils obtiendront.

J'ai pensé pouvoir et devoir dans cette circonstance attirer l'attention sur quelques citoyens que leur empressement à se conformer à la loi et le civisme qui règne dans leurs lettres d'envoi nous fait distinguer. Ces citoyens sont:

Jean Baptiste Navailles, propriétaire de l'habitation Navailles, quartier de Montrouys, représenté par le citoyen Girodon;

François Maillat, propriêtaire de l'habitation Maillat et Roche, même quartier, représenté par le citoyen Ganipel.

Et enfin, la famille Pélevin, propriétaire d'habitations, situées aux Cayes, fond de l'Isle à vache.

Je vous demande pour eux toutes l'accélération qui dépendra de vous relativement à la levée des séquestres qui auraient pu être mis sur leurs propriêtés et à la liquidation des revenus à la restitution desquels ils ont droit.

Une seconde lettre ${ }^{93}$ est enfin adressée uniquement à Sonthonax;

Paris, le 26 pluviose, an IV de la République Française, une et indivisible.

Turben, au citoyen Sonthonax, agent particulier du Directoire exécutif, aux Isles-sous-le-Vent.

Citoyen,

Vous avez bien voulu me promettre que prenant en considération le civisme du citoyen Navailles-Bonnas, propriétaire d'une habitation de son nom, quartier de Montrouys et son exactitude à fournir les preuves de sa résidence, vous feriez user de célérité dans la levée du séquestre qui pourrait avoir été mis sur ses propriétés et dans la liquidation pour la restitution des revenus perçus pendant sa durée.

Trouvez bon qu'ainsi que nous en sommes convenus, j'adresse cette lettre pour vous au citoyen Girodon, son fondé de pouvoir à l'effet de vous rappeler votre promesse qui, vu l'importance et la multiplicité de vos occupations, pourrait n'être point présente à votre mémoire.

Je brûle d'apprendre que votre traversée a été aussi heureuse que je le désire et qu'il est à désirer pour le bien de la colonie.

Salut et fraternité.

33. Papiers Navaille-Bonnas. 
En l'an V, le citoyen Navailles-Bonnas (Jean-Baptiste) reçoit du ministre de la Marine et des Colonies un nouveau certificat de résidence en France. Navailles-Bonnas est toujours porté sur la liste des citoyens restés fidèles à la patrie. "Je vous in vite, dit la lettre $^{94}$ à m'indiquer la colonie où sont situées vos propriétés, ainsi que le nom de votre gérant."

Tout est donc mis en œuvre afin que Navailles-Bonnas ne souffre pas trop longtemps du séquestre. Mais quelle est la situation de l'habitation durant ces années?

Nous n'avons pas trace de correspondances entre Terrien et Navailles de 1794 à 1796.

C'est seulement par une lettre de mai 1796 que Terrien aîné donne de ses nouvelles de Savannah et les lettres de 1796 et 1797 vont nous apprendre quelle a été la situation à l'Arcahaye et sur la plantation durant l'occupation anglaise.

La première lettre est du 31 mai 1796. A Savannah, Terrien est inquiet sur son sort futur. Il vient de recevoir des lettres de son frère. Ce dernier vient de passer six semaines sur la plantation Navailles, sa santé est délabrée et il a dú faire deux voyages. L'habitation est en bon état; les nègres continuent à travailler avec ardeur, mais cette situation ne semble pas devoir se prolonger.

Le revenu de 1795 a été de 41 milliers de café et le résultat du compte de l'année de 2.000 livres en faveur de Navailles. Maigre résultat en vérité, mais qui s'explique si l'on songe aux détournements continuels des nègres qui rendent les récoltes très médiocres et qui nécessitent de nouvelles dépenses.

Terrien aîné dépeint sa propre situation sous un jour très noir; il doit exagérer, car si nous en croyons Girodon, il mêne une vie facile et il a de l'argent. Il s'agit pour lui d'apitoyer sur son sort le lointain propriétaire susceptible de réclamer des comptes précis. Il pense s'établir à Charleston dans la Caroline du Sud et il offre ses services à Navailles et à ses amis.

Des nouvelles plus précises sur la situation de la colonie et de la plantation seront données à Navailles par la lettre du 20 janvier 1797.

Les intérêts de Navailles sont toujours entre les mains de

94. Papiers Navailles-Bonnas. 
Terrien jeune, mais les revenus deviennent de plus en plus modiques et il est difficile de les faire parvenir en France. Le sequestre mis sur les biens n'arrange pas les choses, le désordre est général:... "Là comme ailleurs, en Amérique, comme en Europe, il se passe d'étranges choses. Pillagi! semble être devenu le mot de ralliement de tous les partis, de tous les pays, au moins de la grande majorité des individus" écrit Terrien aînê5.

Le précis historique ${ }^{96}$ comme les lettres de Terrien nous donne des renseignements intéressants. "Dès le 24 juin 1795, dit le précis historique, en exécution d'un acte du Parlement d'Angleterre... le général Williamson avait réglé que les biens des absents seraient administrés par des proposés du gouvernement anglais.

Le 26 septembre suivant, par une nouvelle proclamation, il avait distingué les absents valablement représentés de ceux qui ne l'étaient pas et il voulait que les biens des premiers continuassent d'être gérés par leurs fondés de procuration à la charge d'en rendre compte à l'administrateur général.

Les biens des absents qui justifieraient de leur résidence en pays anglais, neutre ou ami furent affranchis de ce séquestre... La paroisse de l'Arcahaye résistait à l'exécution de ces ordonnances..."

De son côté, Terrien décrit la situation à l'Arcahaye durant l'occupation anglaise à son maitre en France, dans des termes à peu près semblables, mais cependant avec plus de précision.

Les Anglais, dès qu'ils se sont emparés de l'Arcahaye, fait connaitre le Procureur, ont pris une ordonnance séquestrant revenus et propriétés des absents qui ne prouveraient pas leur residence en pays anglais, allié ou neutre. Cette ordonnance dépossédait même les procureurs fondés. Cette clause ayant fait murmurer les procureurs qui menaçaient de quitter la colonie si on détruisait les seules causes qui les y retenaient, l'ordonnance fut remplacée par une autre avec des changements. Mais l'esprit même de l'ordonnance n'était pas changé, l'Anglais maintenant la séquestration des revenus, mais les habitations étant laissées à ceux qui avaient des

95. Correspondance Navailles-Bonnas.

96. Précis historique des Annales de la Colonie française de Saint-Domingue, depuis 1789 à l'année 99. Manuscr. anonyme - $\left(2\right.$ vol. in. $\left.8^{\circ}\right)$, Arch. de la Vienne dŚpôt 102. 
pouvoirs en règle des propriétaires. Ces procureurs devaient verser les produits à des époques fort rapprochées dans les caisses d'administrateurs nommés à cet effet.

Cette ordonnance, déclare Terrien, eut son effet dans toutes les paroisses possédées par les Anglais, excepté l'Arcahaye, où elle manque le sien en partie. Les intrigants, les protégés des Anglais n'étaient pas contents. Aussi décidèrent-ils d'affermer toutes les habitations des absents. Mais ces habitations ne furent pas affermées régulièrement, c'est-à-dire par criées. Elles furent affermées par des intrigants à d'autres intrigants, en sorte que telle sucrerie de 100.000 livres de revenu net est affermée pour 50 ou 55.000 livres. C'est ainsi que Terrien signale trois sucreries valant 150.000 livres chacune affermées toutes trois 150.000 livres.

Un M. Esten ${ }^{97}$, secrétaire du général anglais, commissaire départi pour cette opération, s'adjuge trois sucreries. D'après l'ordonnance, il n'aurait pu en avoir qu'une seule et même aucune suivant l'esprit de l'ordonnance et en raison de sa qualité.

Quelle va être la conclusion de ce pillage pour les propriétaires?

"Les sucreries de l'Arcahaye ont seules provoqué cette opération par leur importance", mais la loi étant générale "elle y a englobé les caffeteries, accessoire modique par leurs revenus amoindris et réduits à moins encore par les dépenses extraordinaires, impositions qu'on établit toujours en raison du nombre des nègres et non en raison de leurs revenus actuels ${ }^{98}$."

Pour l'habitation Navailles, Terrien jeune se présente comme fermier:

..Mon frère a eu la bonne idée, comme je m'y attendais, de se prêsenter pour fermier, surtout pour que les malheureux nègres à qui nous avons inspiré beaucoup d'attachement, de considération, de confiance, n'eussent point affaire à des visages nouveaux.

L'opération annoncée, mon frère se rend vite sur les lieux et voicy le résultat du compte qu'il m'en rend par sa lettre du 9 novembre. Je vous ferai grâce de toutes les indignités et infamies qui précédèrent et eurent lieu dans le cours de cette

97. A l'Arcahaye, le secrétaire du commandant en chef, James Faten, fit rendre le 14 aout 1796 , une ordonnance qui mettait a ferme pour 7 ans, tous les biens d'absents, sans distinction.

98. Correspondance Navailles-Bonnas. 
infâme opération où assistèrent toutes sortes de commissaires et un essaim d'aspirants à des fermes. L'esprit et la lettre même de cette ordonnance favorisant les parents présents des propriétaires absents, il demanda la ferme des mineurs Seguineau pour M. d.d.L.r. qui est sur les lieux, celle de l'habitation Maillat et Roche pour M. Comte, parent du premier et fit valoir de fortes raisons pour demander la ferme des autres habitations pour nous-mêmes. Toutes étaient promises et arrêtées d'avance; on le dit positivement à mon frère et il n'y eut d'amendement qu'en raison de la manière vigoureuse dont il parla. Tant il y a que ...l'habitation Pierre Seguineau a été affermée 15.000 livres (argent de SaintDomingue, bien entendu), au colonel Joynson, anglais.

Maillat et Roche, 12.000 livres au colonel anglais Alen.

Jean Seguineau, 6.000 livres à un officier français homme d'honneur, habitant de Saint-Domingue, qui mérite beaucoup par tout ce qu'il a fait pour son pays; mon frère a taché en vain de faire avoir celle-là à M.d.d.L.r. encore.

Celle des mineurs Seguineau lui a été donné à 7.000 livres, enfin mon frère obtint la vôtre à 5.000 livres, quoique promise à un M. Forbis, frère d'un négociant à la Jamaĩque, à qui on insinua de se désister de ses prétentions, par suite du mécontentement que témoigna hautement mon frère à qui son concurrent offrit un partage ou société que mon frère rejeta et il l'eut seul.

Ces fermes sont pour cette année, terme insignifiant $t^{99} .$. grands événements qui surviendront bien avant ce laps de temps écoulé $e^{100}$.

A cette date, Terrien jeune a donc pu sauver l'habitation et il en demeure le fermier. Un document ${ }^{101}$ particulièrement intéressant, va, par une analyse minutieuse, nous renseigner sur la situation de l'habitation Navailles, en 1796, c'est-à-dire sous l'occupation anglaise.

Ces documents consistent en un bail et en deux inventaires. Bail et inventaire nous donnent d'abord le nombre des nègres restés sur la plantation, ainsi que leur nom et leur origine. C'est tout le problème de la main-d'œuvre qui se trouve ainsi posé à une date où la situation est fort confuse à l'Arcahaye.

99. Illisible dans le texte.

100. Correspondance Navailles-Bonnas.

101. Dépôt des Papiers publics des Colonies, Saint-Domingue. Domaines, administration anglaise. Archives de la France d'Outre-Mer, C 8 D 1 . 
A la veille de la Révolution, le nombre des esclaves a varié de 148 à 150. En 1796, il n'est plus que de 122, se décomposant ainsi:

Nègres............ 43

Négresses........... 64

Négrillons........... 4

Negrittes........... 11

122

L'habitation étant proche des camps, il est certain que plusieurs nègres doivent être occupés aux corvées fort nombreuses.

L'état-civil de tout l'atelier, âge, race, emploi, figure dans les documents.

Les créoles sont au nombre de 50. La proportion des diverses races est la suivante:

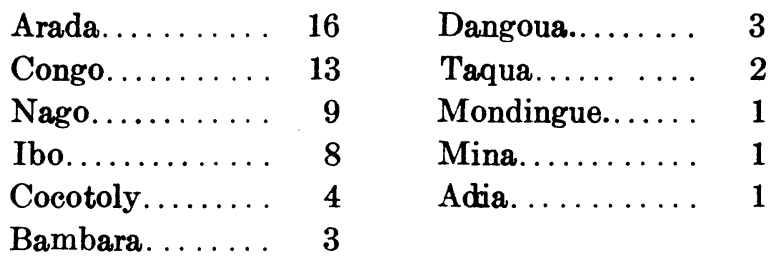

Les éléments de l'atelier sont assez variés. La race dominante est celle des Arada et des Congo. Ces races dominaient sur les plantations du 18e siècle. Les Nagos, Cocotolys et Arada venaient de la Côte-d'Ivoire, le grand pays de traites pour Saint-Domingue. La plupart d'entre eux devaient avoir été achetés avant 1790.

Pour connaître la force réelle de l'atelier, en 1796, il nous faut examiner l'âge des noirs, la proportion des noirs âgés de moins de 20 ans, des noirs âgés de 20 à 50 ans, des vieillards.

NOIRS AGES DE MOINS DE 20 ANS

$\begin{array}{rccc}\text { AGE } & \text { Hommes } & \text { Femmes } & \text { Total } \\ 0-5 & 3 & 8 & 11 \\ 5-10 & 2 & 2 & 4 \\ 10-15 & 5 & 5 & 10 \\ 15-20 & 2 & 5 & 7 \\ & \overline{12} & -20 & 32\end{array}$


NOIRS AGES DE 20 à 50 ANS

20-25

$25-30$

30-35

$35-40$

$40-45$

45-50

3
2
5
2
3
5

20

$\begin{array}{rr}14 & 17 \\ 1 & 3 \\ 1 & 6 \\ 5 & 7 \\ 2 & 5 \\ 9 & 14\end{array}$

32

17

3

6

52

7

5

14

NOIRS AGES DE PLUS DE 50 ANS

50-55

au delà de 55
2

14

16

48

\begin{abstract}
2
\end{abstract}
20

22

74
4

34

38

TOTAL GENERAL

74

122

Le nombre total de 122 ne doit pas faire illusion. Sur 122 noirs, il n'y a environ que 22 hommes et 37 femmes en état de travailler et encore faudrait-il déduire de ce chiffre quelques infirmes. Il n'y avait pas plus d'une quarantaine de travailleurs en 1796 sur l'habitation Navailles. Et les espoirs de renouveler l'atelier, sans faire appel à une main-d'œuvre étrangère étaient assez minces: 10 hommes et 16 femmes en état de procréer étaient seulement présents sur la plantation. Quand on connait aux Antilles le pourcentage de la mortalité infantile, il est permis de dire que l'atelier ne pouvait se renouveler de lui-même.

Dans la liste des nègres figurent quelques spécialistes: d'abord les deux commandeurs, un jeune charpentier créole, un cuisinier congo. C'était ceux d'ailleurs dont le prix d'estimation était le plus élevé, de 1500 livres à 2500 livres, en 1796. Les esclaves dans la force de l'âge sont estimés à la même époque, de 1000 à 2000 livres. Ce sont les créoles qui sont estimés le plus cher. Les places de confiance sont occupées par des créoles, notamment celles de com- 
mandeurs. Au delà de 60 ans, les prix sont dérisoires et tombent à 5 livres.

Entre 1788 et 1796, soit presque dans l'espace de 10 ans, l'effectif n'a baissé que de 26 unités. Mais en fait, il faudrait connaître l'âge des noirs présents sur la plantation en 1788, pour tirer une conclusion, car nous avons affaire à un atelier qui a beaucoup vieilli et qui ne s'est que très peu renouvelé. Très vraísemblablement, la quarantaine de vieillards que nous trouvons en 1790 vivait déja sur la plantation en 1788 .

La diminution de l'effectif peut s'expliquer par la mortalité et la dénatalité, mais nous n'avons pas d'éléments de comparaison entre 1788 et 1796 et nous ne pouvons émettre que des hypothèses.

Pour la culture, le bail nous donne la situation de la plantation en 1796. Par comparaison avec 1788, la situation en 1796 est lamentable.

Il y a seulement 10 carreaux plantés et 100 carreaux qui étaient auparavant en culture sont maintenant en halliers. Le revenu possible, d'après les experts qui dressent l'inventaire, n'est plus que de 20 milliers de café. Mais la culture nécessite autant de frais que si elle rapportait 100 milliers de café. N'oublions pas qu'avant la Révolution, la plantation Navailles rapportait dans les 80 à 85 milliers de café et que, même Therrien avait intérêt, en 1796, à déprécier la valeur de la terre. Toujours est il que les revenus sont infimes; la main d'œuvre, comme nous venons de le voir, très faible, ne peut suffire à l'exploitation de la terre.

De plus, l'habitation est exposée aux brigands, il faut du personnel pour se tenir sur la défensive et la proximité des camps entraîne des corvées nombreuses d'où un appel à la main-d'œuvre noire, main-d'œuvre qui fait défaut à la culture.

La situation n'est guère brillante pour la maison Terrien frères et Castillon ${ }^{102}$ qui afferment la plantation pour 7 ans, du 24-X-96 au 24-X-1803, au prix de 5.000 livres par an, somme dérisoire.

L'estimation des meubles, des animaux et des bâtiments fait aussi ressortir une situation peu brillante.

102. Il s'agit de Castillon-Dyperron, fils d'un négociant bordelais, associé a Terrien, qui écrivait encore à $M$. de Navailles, en l'an VII (cf. lettre de Navailles au Chef de Batailles Navères - 15 pluviose, an X - Domaines C. 43/D 33). 
Voici ce qu'énumère l'inventaire:

\section{MEUBLES}

8 grands fauteuils garnis en cuir.

2 canapés dans le plus mauvais état.

2 tables marbres avec leur pied doré.

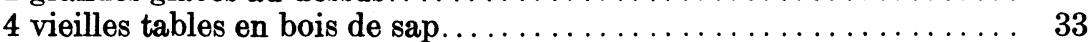

2 vieilles armoires très mauvais état. . . . . . . . . . . . . . . . . . 100

3 bois de lit en bois de sap............................

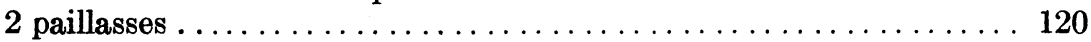

1 mauvais matelas.

1 vieux bureau en bois du pays....................... 20

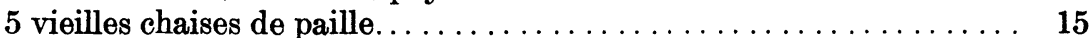

7 bats (?) en mauvais état.............................. 100

36 sacs en médiocre état............................... 144

2 vieux moulins incapables de servir................... 180

Ce mobilier parait assez simple. La plupart des objets sont en "bois de sap", quelques-uns en bois du pays et l'ensemble laisse l'impression d'une vie assez rustique.

Les animaux ne sont guère nombreux en 1796; il reste 6 mulets en bon état, estimé chacun 500 livres et 7 mulets vieux estimés 200 livres, plus un vieux cheval, estimé 200 livres.

Les bâtiments comprennent:

\section{BATIMENTS}

1 grande case ( 9 chambres ou cabinets) couverture mauvais état. . . 8.000

1 hôpital avec la cuisine, ensemble. . . . . . . . . . . . . . . 2.000

1 petit pavillon en maçonne. . . . . . . . . . . . . . . . . . . . . . 800

1 magasin à café. . . . . . . . . . . . . . . . . . . . . . . . 3.300

1 magasin à grain, couverture mauvaise. . . . . . . . . . . . . . 1.500

1 moulin octogone. . . . . . . . . . . . . . . . . . . . . . . . . . . 5.000

4 cases à nègres (grandes et petites) . . . . . . . . . . . . . . 6.000

1 case à l'économe. . . . . . . . . . . . . . . . . . . . . . . . . . . . 1.000

1 écurie "en maçonne plaine" ....................... 2.000

La grande case était importante puisqu'elle comprenait 9 chambres ou cabinets, mais la couverture en mauvais état laisse supposer que des réparations n'avaient pas été faites depuis longtemps. L'économe gérant avait sa case. Pour les noirs, 4 cases, grandes et 
petites, un hôpital avec cuisine. Enfin, pour l'exploitation de la plantation, un moulin, un magasin à café, un magasin à grain et pour les animaux, une écurie.

Cette énumération nous laisse l'image d'une plantation qui dut être importante.

La conclusion de l'inventaire de 1796 est fort pessimiste. Il faut entretenir un grand nombre d'employés pour maintenir les nègres et les mettre en état de défense contre ceux qui sont insurgés.

Le dépérissement de la plantation est inéluctable ${ }^{103}$ car la terre se dégrade chaque jour, les cafés dépérissent annuellement sans qu'on puisse les remplacer.

Terrien reste cependant par nécessité optimiste. Ne se félicite-til pas d'avoir sauvé la plantation et d'en demeurer le fermier. Les deux frères prennent bien soin de faire savoir au propriétaire qu'ils ne poursuivent aucun objet de spéculation.

Pourtant "celle ferme, quoique seule nous aidera nous aidera un peu dans nos dépenses icy et il pourra en revenir quelque chose à $M$. de Navailles ${ }^{104}$."

On ne promet pas grand'chose au propriétaire, n'a-t-on pas essayé de sauver tout ce qui pouvait être sauvé et n'est-il pas heureux qu'un Terrien ait pu se maintenir à la tête de l'habitation? Que serait devenue l'habitation si d'autres eussent été fermiers? D'autant plus que Terrien jeune qui était procureur d'autres habitations n'a pu conserver que l'habitation Navailles. Les revenus évidemment seront modiques et les dépenses étant donné les temps actuels plus ou moins fortes. D'autre part, Terrien aîné conseille à son maître de se faire délivrer un certificat de résidence en pays allié ou neutre et non en guerre avec l'Angleterre, car l'ordonnance est toujours maintenue qui dit que les habitations et les revenus

103. La plantation de nos jours, d'après une lettre du frère Lucien-Joseph du Collège Saint-Louis, à Port-au-Prince, qui, à notre demande, a fait une excursion sur les lieux mêmes où s'élevait au 18ème siècle, la caféière Seguineau-Navailles, n'est plus qu'une région abandonnée et sans culture. "Cette région que l'on soupconne avoir été très riche est en marche vers la désolation," écrit-il.

"Des ruines occupent la crête (1120 mètres)". Une chapelle a été édifiée sur l'emplacement de l'habitation et cette "chapelle, le presbytère, les dépendances ont été édifiées au dépens des pans de murs qu'on a trouvés sur les lieux." (Lettro du 25 juin 1951). Quelle désolation, sur cette terre riche et fertile, laissée à l'abandon depuis l'occupation anglaise jusqu'd nos jours!

104. Souligné dans le texte. 
seront laissés à la disposition des procureurs qui justifieront de la résidence des propriétaires en pays allié ou neutre. Terrien enfin s'efforce de se justifier au sujet des récoltes antérieures, vis-à-vis des accusations de Girodon, le gérant, d'autant plus que Navailles veut être renseigné exactement, sa situation financière en France semble à cette époque assez difficile. Il a demandé des précisions à Terrien sur les 2.000 livres de l'année 1795 et il s'étonne que ce dernier ne parle pas de la récolte de 1797. Or, la récolte de 1796 n'a pas été encore faite, mais les 2.000 livres ne sont pas le solde dû de l'année 1795 seulement, mais dû au 31 décembre 1795 et résultat de toutes les années précédentes.

Et Terrien s'engage dans de longues explications assez confuses, mais que le propriétaire en France devra accepter.

(à M. de Navailles-Bonnas, Savannah, 30 janvier 1797 ${ }^{105}$.

Je vous ai envoyé une note des revenus et de leur emploi, de 1791 à 1795, aussi une note de notre position avec l'habitation. Je vous donne de nouveau cette dernière...

Au 31 décembre 1791, il était dû à l'habitation 2.392 livres, porté au compte de 1792 , dont le résultat était 2833 livres dû par l'habitation, portê à celui de 1793, 31 décembre.

A cette époque, l'habitation était débitrice de 2805 livres porté au compte de 1794, dont le résultat était encore 5253 livres, à quoi je dois supposer qu'il y aura quelque chose à ajouter à votre crédit pour 1796; indépendamment de vos revenus, je comptais bien vous faire des remises du prix de plusieurs de vos nègres enrôlés dans les corps noirs qu'on devait payer à raison de 2.000 livres pièces, mais ce paiement a été éludé à l'aide de divers prétextes.

Débiteur ou créditeur, à mon arrivée sur ce continent, je vous aurais envoyé quelques secours si j'avais été en situation de pouvoir le faire. Mais quelques moyens m'étant parvenus au moment où votre dernière lettre est venue me témoigner vos besoins urgents, je me suis immédiatement décidé à l'envoi d'un petit secours... ayant été bien embarrassé pour vous le faire parvenir sûrement... Aussi ai-je saisi avidement l'occasion rare d'un bâtiment directement d'icy pour Bordeaux, que je fais consigner à M.B.R.Y. et expédier par un négociant de ma connaissance, M. William Belilar: je lui ai compté icy 500 piastres gourdes ${ }^{106}$, pour vous

105. Correspondance Navailles-Bonnas.

106. La piastre gourde vaut 8 livres 5 . 
être payées à Bordeaux, en 500 piastres gourdes d'Espagne ou autres, monnaie d'or ou d'argent à votre satisfaction. Je joins icy son obligation assez bien motivée, je crois, pour n'avoir aucun schisme à craindre. Il doit me produire votre reçu dans six mois, mais je désire que vous soyez le premier à m'annoncer réception de cette petite somme. Elle serait du double, du triple plus fort, si je ne consultais que mes désirs. Mais voicy en peu de mots ma situation icy. Une famille assez nombreuse à soutenir, point d'autres moyens que le travail de mes nègres, dont j'ai déjà perdu deux, morts, un troisième péri dans un incendie qui a eu lieu icy, le 26 novembre qui a détruit la moitié de cette ville et un magasin de marchandises que je fais tenir où j'ai sauvé beaucoup et perdu pour 700 gourdes, beaucoup aussi en raison de ma situation actuelle.

Du reste, de belles espérances d'une habitation encore toute neuve à Saint-Domingue, de mes travaux sans relâche, au moyen de capitaux assez considérables que mes amis mettent en refuge dans mes mains, en me disant de les faire valoir pour notre avantage commun. Cette position trop vraie ne me permet pas de suivre mon inclination nouvelle.

En attendant, à pouvoir faire encore quelque chose de plus pour vous, Monsieur, il serait d'une satisfaction bien douce pour moi, de savoir que ce petit secours vous en ait fait éprouver. Il vous va par, la goëlette Salty de Boston, capitaine Lewis, expédié de Savennah, par M. William Belilar, signataire de l'obligation ci-jointe. Par ce même bâtiment, j'expédie la première lettre.

Quelques mots ajoutés dans cette missive sur Sonthonax et Raymond qui confirment le point de vue des Terrien à l'égard des réformes accomplies par ces derniers dans l'île. Sonthonax a aboli l'esclavage le 29 aout 1793, et Terrien, comme beaucoup d'autres, considère toujours le maintien de l'esclavage comme le fondement de la propriété a Saint-Domingue. D'ailleurs, les colons blancs de Saint-Domingue, bannis, déportés, expatriés et réfugiés aux ÉtatsUnis, n'ont cessé de protester contre Sonthonax "ce délégué parjure ${ }^{107} . "$

Et Terrien est outré de ce que Navailles ait confié ses intérêts à Sonthonax:

107. Archives Nationales A.F. D XXV, 59, 69. 
Vous parlez de vos intérêts recommandés aux commissaires $\mathrm{S}^{\mathrm{x}}$ et $\mathrm{R}^{\mathrm{d} 108}$. Dieu préserve vos propriétés et vos personnes d'avoir rien de commun avec ces individus ou autres qui peuvent leur ressembler! C'est assez vous en dire.

Terrien termine enfin sa lettre comme toujours par des paroles d'espoir. Ne fut-il pas d'ailleurs toujours optimiste, même dans les mauvais moments!

Ce que nous vous dirons de plus consolant pour le moment, c'est que par la situation actuelle des choses à SaintDomingue, il y a peu d'appréhensions à avoir pour la destruction de vos propriétés et que si la paix rendait demain ce pays à la France, et que la France y envoyât des gens qui voulussent réellement conserver, elle serait conservée et quoique sans terre vierge encore, pourrait être remise en etat de vous donner de belles rentes. Quelque ingrat que soit le travail de régénérer de vieilles terres dans les cafféteries, nous l'entreprendrons avec plaisir et suecès dans la partie basse de votre habitation, son local aidant naturellement à ce travail.

Messieurs Seguineau neveux ont deux habitations contiguës, une assez jeune encore, une autre absolument neuve, toutes deux ensemble d'un produit immense qui a dû laisser à Messieurs Magnas de grands moyens de leur faire des remises.

Je crois n'avoir plus rien à vous dire et pouvoir terminer icy cette longue conversation...

- P.S. 30 janvier.

J'ai des lettres de mon frère, jusques au 28 du mois dernier; il ne me dit pas un mot des habitations, signe qu'il n'y a rien de nouveau absolument.

Le capitaine Lewis (prononcer Louis) part demain. II est charge d'acquitter l'obligation ci-jointe. Puisse-t-il vous l'apporter en quarante jours, vous trouver en bonne santé et me rapporter bien vite de vos nouvelles.

En somme, l'habitation Navailles ne semble pas avoir trop souffert de 1793 a 1798, pendant l'occupation anglaise. Si les revenus sont à peu près insignifiants, le propriétaire peut se consoler en songeant que ses biens continuent à demeurer en sa possession, grâce aux frères Terrien. Cependant, si le capital est intact, les re-

108. Soathonax et Raymond. 
venus seraient les bienvenus pour Navailles qui est dans une position financière précaire.

Cette période voit la demi-désorganisation de tous les ateliers. Mais on tenait tant bien que mal. On se soutenait pour atteindre la paix générale. La fièvre jaune et Toussaint-Louverture harassèrent les Anglais. Le général Maitland, commandant en chef, reçut des instructions précises pour traiter avec Toussaint et Rigaud, alliés aux Anglais ${ }^{109}$. La convention d'évacuation ratifiée par Toussaint, le 2 mai 1798, remettait entre ses mains tous les quartiers de l'ouest qui avaient jusque là résisté à ses attaques, dont l'Arcahaye. Le 9 mai, Dessalines, un des généraux de Toussaint, prenait possession de l'Arcahaye. L'administration des biens d'absents passe aux mains des agents de Toussaint et la plantation Navailles fut séquestrée.

C'est la dernière image que nous ayons de la plantation. Nous sommes en 1798; la question de Saint-Domingue est loin d'être réglée et c'est au fils de Jean-Baptiste de Navailles-Bonnas qu'il appartiendra de liquider la situation. Pour lui, un seul problème se posera: celui de l'indemnité à toucher. La France aura alors abandonné une magnifique partie de son empire colonial aux Antilles.

\section{Chapitre SIXIÈme}

\section{LA QUESTION DE L'INDEMNITE}

Une grosse lacune apparaît dans la documentation, à partir de 1797, jusqu'en 1823, c'est-à-dire jusqu'au moment où se pose la question de l'indemnité.

Une lettre datée de Bordeaux, du 6 septembre 1825, adressée à Navailles-Bonnas, à Larreule, par sa sœur, Mme de Roll, définit assez bien l'état d'esprit des anciens propriétaires de Saint-Domingue:

Je n'entends parler d'autre chose ici, mon cher ami, que de ce que les propriêtaires colons espèrent retirer de l'indem-

109. Montrouis est évacué par les Anglais en décembre 1797. - Précis historique des Annales, ms. I, 302. 
nité de Saint-Domingue, au point que j'en ai la tête tournée. Les uns ont de hautes prétentions, les autres ne comptent que sur très peu de chose. Dans tous les cas, il faut toujours se mettre en règle, en réunissant autant que possible, toutes les pièces qui assurent nos droits. D'abord le testament de mon oncle qui doit désigner sous quel nom l'habitation léguée à ma mère était connue, les comptes que tu peux avoir des revenus des deux années de jouissance qu'elle a eue, de la nature de ses revenus, l'état du nombre de nègres et les autres renseignements extérieurs à la mort de mon oncle... enfin, mon ami, ne néglige rien pour pouvoir faire à temps nos justes réclamations...

Le 21 novembre 1825, le Directeur du bureau créé pour l'exécution de l'ordonnance royale du 1er septembre 1825, demande à Navailles-Bonnas des précisions sur "ses propriétés rurales et urbaines", à Saint-Domingue.

Très suggestive est la copie de la réclamation adressée au Directeur de la Cour des Comptes, de la main même de NavaillesBonnas.

Elle est datée du 7 décembre 1825 et est adressée à $M$. Vante ${ }^{110}$, directeur de la Cour des Comptes, sous le couvert du Ministre des Finances.

Monsieur Joseph Henri Maximilien de Navailles-Bonnas, demeurant à Larreule, canton de Maubourguet, département des Hautes-Pyrénées, a l'honneur d'exposer à Messieurs les membres composant la commission d'indemnité pour les colons de l'île Saint-Domingue, que dame Françoise Séguineau, sa mère, possédait à l'île Saint-Domingue, une habitation avec toutes ses dépendances et la moitié d'une maison en sa qualité d'héritière de feu Monsieur Pierre Séguineau, son oncle paternel, suivant le testament de ce dernier qui a eu lieu sous la date du 18 mai 1789, au rapport de Maître Barberet, notaire à Bordeaux - que ladite dame Séguineau est décédée le 14 juillet 1822, à la survivance de quatre enfans, du nombre desquels est l'exposant, après lui avoir légué par préciput le quart de tous ses biens, suivant son testament au rapport de Maítre Bascle, notaire à Larreule, sous la date du 11 octobre 1813 - que M. Auguste de Navailles-Bonnas, autre fils de ladite dame Seguineau, est

110. Vante (ou Wante) était un ancien ordonnateur de la Marine à SaintDomingue. 
décédé le 17 mai 1825, sans descendans, après avoir disposé de tous ses biens en faveur de l'exposant, suivant son testament au rapport de Maître Bascle, notaire, sous la date du 20 août 1822; par cet ordre, l'exposant a droit à la succession de sa mère, savoir: au quart par préciput et ensuite à la part cohéritière et à celle qui revenait au dit feu $\mathrm{M}$. Auguste de Navailles-Bonnas, son frère.

A l'appui de sa demande, il présentera:

1o - le testament de feu M. Pierre Seguineau;

20 - l'acte de décès de feue dame Seguineau, sa mère;

3o - le testament de la dite dame Seguineau;

40 - un acte de notoriété qu'il était le fils et héritier de ladite dame Séguineau.

L'exposant désirant profiter du bénéfice de l'ordonnance royale sous la date du ler septembre 1825, forme sa demande pour obtenir l'indemnité qui pourra lui revenir sur les biens que feue sa dite mère possédait dans l'île Saint-Domingue, en vertu des titres plus haut relatés dont le détail suit:

1o - l'habitation est située dans la partie de l'ouest de l'île de SaintDomingue;

20 - elle est sise à la Nouvelle Saintonge, paroisse de l'Arcahaye;

3o - elle est connue sous le nom de Pierre Seguineau aîné;

40 - il réclame comme héritier de dame Françoise Claire Séguineau.

5o - le gouvernement concéda aux Seguineau frères il y a quatrevingts $a^{111}$ une grande partie de terrain, c'est là que se formèrent les quatre habitations de la famille dont la précitée fait partie, on ignore le nombre de carreaux de terre et ses abornemens.

60 - L'habitation est une caffeyère.

70 - L'atelier se composait, en 1791, de cent cinquante nègres travaillant;

80 - On ignore la nature des moulins;

90 - Il y avait sur l'habitation en 1791 soixante mulets.

10o - On ignore le nombre des cabrouets à mulets ou à boufs.

110 - On ignore le nombre des bêtes à corne.

120 - L'habitation a produit, en 1789 , cent vingt cinq mille trente deux franes d'après les lettres de Terrien frères, chargés de procuration de Françoise Claise Seguineau à Saint-Domingue. On porte la valeur de l'habitation à huit cent mille francs.

13o - Le magasin ou l'embarcadère, servant d'entrepôt pour les caffés de l'habitation précitée est situé au lieu du Monrouis. On ignores sa distance de l'habitation.

111. Soit vers 1745. 
140 - Les documents consistent dans le testament précité de feu Pierre Seguineau aîné et dans les lettres de M.M. Therrien frères, chargés de procuration pour ladite habitation à Saint-Domingue.

Feu Pierre Seguineau a aussi donné et légué par ledit testament précité à Françoise Claire Seguineau, épouse de M. Jean Baptiste de NavaillesBonnas, la moitié de la grande maison qui est située vis-à-vis des prisons de la ville de Saint-Mare, commune et indivise entre lui testateur et les enfans de feu Pierre Seguineau jeune décédé, secrétaire du roi.

Navailles-Bonnas,

à Larreule, canton de Maubourguet, département des Hautes-Pyrénées, ce 9 septembre 1825.

La valeur de la plantation, d'après Navailles-Bonnas, paraît légèrement gonflée. Un capital de 800.000 francs rapportant 125.032 francs, cela donnerait environ un intérêt de $15 \%$.

Dans une seconde lettre, adressée toujours à M. Vante, Navailles-Bonnas précise le montant annuel en denrées: "an 1788: 85.832 milliers de café - en 1789: 95.322 milliers de café" - ainsi que le nom sous lequel l'habitation était connue: "Sous le nom de Pierre Seguineau aîné, jusqu'en 1789, époque de sa mort, puis sous celui de Navailles-Bonnas, parce qu'elle fut léguée par Pierre Seguineau jeune, à sa nièce, Claire Françoise Seguineau, épouse de M. Navailles-Bonnas". Il ajoute: "on ne connaît ny les abornemens ny les noms des voisins, mais on pense que les voisins sont les trois autres habitations Seguineau."

Navailles-Bonnas renonce, en 1826, à se rendre lui-même à Paris comme il avait pensé le faire un moment et charge de ses intérêts MM. de Jonquière et Augé, dont l'un avait été chef de bureau au ministère de la Marine. "La commission est de $2 \%$ de ce qui reviendra aux colons", qui chargeront ces derniers de leurs intérêts.

Pour obtenir plus facilement satisfaction, Navailles fait appel a son ami, M. Ducasse de Horgues, député des Hautes-Pyrénées, son "ami intime", lié avec plusieurs membres de la commission.

En 1827, une grave confusion se produit. Les fondés de pouvoirs sont informés que, se basant sur les archives de Saint-Domingue, une Mme veuve Seguineau, à Paris, a également des droits. En réalité, la preuve se fait bientôt qu'il s'agit d'autres Seguineau, étrangers aux Seguineau-Navailles. 
En 1827, toujours, de nouvelles difficultés. On exige l'acte de décès de Pierre Seguineau. De Navailles écrit: “...Je n'ai que les lettres des frères Terrien... fondés de ma mère à Saint-Domingue... sur la quantité de revenus que ferait l'habitation dans les années les plus rapprochées de celle de $1789 . .$. j'ai oul dire qu'ils étaient morts aux Etats-Unis, en $1794^{112}$; mais encore veut-on que je fasse légaliser leur signature, chose impossible..."

Des difficultés de famille également, le cousin de Guilhemauson, de Bordeaux, demande 1.000 frs. en sus des dixièmes de la créance, même davantage d'après une lettre de Seguineau'Lognac qui, cousin lui-même de Navailles, semble s'être entremis entre les deux parties: “...J'ai vu M. de Guilhamauson... il élève prétentions en capitaux et intérêts à 57.492 , savoir: 26.133 de capital et 31.359 francs les intérêts, qu'il fait partir de la paix d'Amiens. D'après eux, le 10 e en vertu de la loi, représenterait la somme de 5.749 francs... vous êtes passible de ce droit sur vos biens de France ...si vous voulez en finir pour six mille francs, il pourrait y souscrire afin d'éviter toute discussion ultérieure..."

Le 7 février 1827, l'affaire n'est guère avancée. De Jonquière et Augé, chargés des intérêts de Navailles-Bonnas font connaître le numéro d'inscription de la demande d'indemnité, c'est 3.145. "L on en est à peine au no 1450 ... nous craignons comme vous que le gouvernement d'Haïti ne paye pas les 4 derniers cinquièmes de l'indemnité; mais si cela a lieu, nous espérons que le gouvernement français y suppléera en tout ou en partie."

Le 23 mars 1828, l'intervention du préfet des Hautes-Pyrénées, M. Johan, qui informe Navailles-Bonnas qu'après bien des perquisitions, il a découvert à Nantes Mme Fleury, sœur des frères Terrien, morts l'un près Jérémie, à Saint-Domingue, l'autre aux Etata-Unis; "elle-même aurait besoin des extraits mortuaires que vous désirez". Mais M. le Préfet ajoute, sur un petit carré de papier: "Je crois que Mme Terrien a les extraits mortuaires, mais qu'elle a peu de réclamations contre la fortune de ses frères. Ceci entre nous..."

La situation est plus claire le 5 mai 1828 et s'avance vers une solution.

112. Il s'agit des frères Terrien, au moins de l'un d'eux. Inexact sans doute, puisque la dernière lettre de Terrien aîné est de 1797 et il parle encore de son frère. 
A cette date, de Jonquières et Augé écrivent: Nous avons reçu le 2 de ce mois les conclusions des commissaires du roi et du secrétaire général, sur nos demandes en indemnité; qui sont de fixer la valeur des propriétés de M. Seguineau, à 498.842 frs. 67 c. dont le dixième formerait l'indemnité...

Le commissaire admet comme constantes les assertions de M.M. Terrien frères, c'est-à-dire que l'habitation avait, en 1790; 150 noirs et qu'elle avait produit en trois ans 246.088 milliers de café. Mais elle n'a aucun égard au haut prix auquel il avait été vendu; en effet ce prix qui a été jusqu'à 27 sous, tandis que pendant la guerre d'Amérique, il ne s'élevait qu'à 7 ou 8 sous, (des colonies), était trop variable pour que la commission ne fixât pas une base qui est un prix moyen entre les cours ordinaires et les plus élevés. En conséquence, elle a statué que quand le produit en nature d'une cafféterie serait connu, l'on compterait le café à 750 frs. le millier. Et comme, en général, ce genre de culture épuisait la terre, elle a déterminé aussi par un réglement que quand le produit serait connu, il serait multiplié par 8 , pour trouver la valeur de l'habitation et c'est le $10 \mathrm{e}$ de cette valeur qui forme l'indemnité.

Ainsi, le tiers de 246.088 étant 82.029 milliers de café, cette dernière quantité est regardée avec raison comme représentant le produit d'une année de l'habitation; à raison de 750 frs. le millier, elle produit 61.522 frs. regardé comme le revenu d'une année également. Cette somme, multipliée par 8, donne pour la valeur capitale: $492.176 \mathrm{frs}$. dont le $10 \mathrm{e}$ est: $49.217 \mathrm{frs}$. 60 c. Si cette propriété était fixée sur le nombre de 150 noirs pour chacun desquels on donne $325 \mathrm{frs}$. (fonds compris), vous n'auriez eu que 48.750 frs.

Quant à l'entrepôt, il paraît que les héritiers des autres Messieurs Seguineau ont produit un inventaire du ler mars 1775, d'après lequel M.M. Pierre Seguineau jeune et Jean Seguineau auraient eu chacun un entrepôt contigu à celui de leur frère et que chaque propriété de cette nature aurait été estimée à 3.500 livres (des colonies), mais il paraîtrait aussi que chaque entrepôt était une dépendance de l'habitation de son propriétaire et ne servait que pour son usage. En conséquence, la commission liquidant votre habitation sur le produit, nous ne pensons pas que les conclusions du commissaire du roi qui sont de ne rien donner spécialement pour l'entrepôt et dele considérer comme une portion de l'habitation, puissent être combattues avec avantage... Nous devons même vous faire remarquer que M.M. Terrien frères n'en font pas mention dans leurs lettres; qu'il ne devait donc être 
d'aucun produit et qu'il est assez raisonnable de le considérer simplement comme une case servant de magasin, située à quelque distance de l'habitation et à portée de la route ou le café pouvait être aisément chargé sur les cabrouets pour être transporté.

D'après la liquidation déjà faite pour les enfans de $M$. Pierre Seguineau jeune ou leurs représentants, le même inventaire a été pris pour base de la valeur de la maison: elle y figure pour une faible somme de 20.000 livres (13.333 frs. $33 \mathrm{c.}$.). Ne pouvant fournir aucune autre preuve, ils y ont adhéré; nous ne pourrions certainement obtenir mieux. Votre moitié serait donc de:

6.666 f. 67 dont le 10 e est............ 666 f. 66 c pour l'habitation. .................49.217 f. $60 \mathrm{c}$

Total de l'indemnité. . . . . . . . . . 49.884 f. 26 c

Cette somme n'est pas forte; mais combien vous êtes plus plus heureux que bien d'autres colons qui n'ont la preuve d'aucune valeur déterminée pour leurs propriétés! Nous sommes convaincus que ce qu'il y a de mieux à faire est d'adhérer aux conclusions prises...

Ducasse, député des Hautes-Pyrénées, et ami de Navailles, annote cette lettre et écrit: "Je suis de l'avis de vos agents. Acceptez l'indemnité telle qu'elle a été liquidée. Il serait impossible de parvenir à avoir davantage."

Dans sa réponse à ses agents, Navailles-Bonnas se rallie à ce point de vue, bien que "les revenus de l'habitation se soient élevés pendant que ma mère en a joui, à un tiers plus haut que la Commission ne les a portés..."

Mais de nouvelles difficultés surgissent encore en septembre 1828: "La commission a prononcé le 3 juillet, sur vos réclamations et a liquidé vos indemnités et celles de Mesdames vos sœurs à 49.884 frs. 26 c. comme l'avaient proposé le secrétaire général et le commissaire du roi...

Hier soir, nous avons reçu un avis du Directeur Général de la Caisse des Consignations qui porte que vos indemnités ne peuvent être payées par suite d'opposition. Probablement, elles sont sous le nom de Seguineau, c'est ce dont nous nous assurerons..."

Enfin, en décembre 1828, les dernières oppositions sont levées. La même année, Navailles-Bonnas et sa sœur Mme de Roll touchent un cinquième de l'indemnité. 
En février 1829, Navailles désire vendre les 4 derniers cinquièmes de son indemnité. Ses chargés d'affaires lui conseillent de ne pas le faire: "Jamais on ne les a vendus au-dessus de $40 \%$ et ce n'a été qu'un moment; bientôt on les a offerts de partout à 35 et puis à 33, et depuis assez longtemps, nous n'avons entendu parlé d'aucune vente. Ces prix sont si bas, que l'un des deux contractants doit faire une mauvaise affaire, soit que le gouvernement paye ou ne paye pas. Aussi, n'avons-nous conseillé à personne de vendre ni d'acheter."

La question des biens de Saint-Domingue étant définitivement liquidée, "un des chapitres les plus intéressants, sinon douloureux, de l'histoire coloniale de la France"113 se terminait. C'était l'anéantissement d'une classe sociale et d'immenses richesses.

Ainsi s'achevait l'histoire de la plantation Seguineau-Navailles. Elle n'avait pas eu un long destin. Mais de 1745, où les frères Seguineau l'établirent, à 1829, où Maximilien de Navailles-Bonnas touche son indemnité, toute symbolique, ces quelques vieux papiers de famille et ces courtes années nous permettent d'aborder plusieurs questions importantes de l'histoire générale des plantations et de la colonie. Elles n'ont pas toutes le même relief, mais toutes nous aident à comprendre mieux ce passé antillais si mal connu.

On a d'abord un aspect de la vie politique; la vue est brève, mais 1790 est pour Saint-Domingue une année décisive, celle où une partie des colons commence à se détacher de la France, pour résister à sa politique favorable, bien timidement pourtant, à l'égalité des couleurs. Les lettres des frères Terrien exposent le point de vue de beaucoup de blancs et marquent nettement la réaction du commerce devant la politique autonomiste de l'assemblée de SaintMarc. Elles offrent quelques éléments pour tenter quelque jour la carte des opinions politiques de la colonie.

Au sujet des esclaves, nous apprenons peu de chose. Il nous faudrait des comptes et des lettres, s'échelonnant sur plus d'années et des états ou des prises de possession qu'on pat comparer. Nous

113. Marcille (A) - L'indemnité aux anciens colons de Saint-Domingue (conférence du 27 mars 1936, au Centre d'Etudes juridiques coloniales). 
n'avons là que des lettres de procureurs. Les Terrien se tiennent comme tels assez loin de la vie quotidienne de l'habitation et des esclaves. Ces témoignages de gérants ou de procureurs sont généralement des témoignages dirigés, soit pour montrer les voleries et et l'incapacité de leurs prédécesseurs immédiats ou pour montrer leur compétence et l'assiduité de leurs soins à eux. Les dires des frères Terrien sont intentionnellement optimistes. Ils veulent aller au devant des on dit qui se colportent en France sur les désordres des ateliers, leur mauvais esprit dû aux mauvais soins et aux négligences des représentants des maîtres. Ils cherchent à rassurer les Navailles-Bonnas prêts à croire les pires nouvelles. Ils sont de ceux qui se persuadent que la peur du désordre provoque le désordre. De ce qu'ils répètent avec tant de complaisance, voyons l'intention. C'est sur l'attitude des Terrien devant le problème de l'esclavage tel que le pose la division des blancs et des mulâtres libres revendiquant leurs pleins droits que leur correspondance sera instructive.

Elle nous explique aussi pourquoi ces plantations coloniales des Antilles étaient fragiles et un vrai paradoxe dès que le maître n'était plus là. Elles n'étaient, plus quand le propriétaire était absent, que des capitaux lointains aux revenus non ou peu contrôlés, donc très aléatoires et irréguliers. Les propriétaires ne pouvaient pas vraiment surveiller leur administration, apprécier l'importance et l'urgence des travaux demandés par leurs procureurs, la valeur réelle des esclaves achetés, les causes des maladies et des décès. Navailles-Bonnas était de ces maîtres qui n'avaient jamais vu leur domaine,... ni même un esclave. Il ne verra jamais de plantation. Après 1793, il n'en aura quasi plus de nouvelles. Les procureurs, mieux les gérants en seront les vrais maîtres, ce qui n'est pas une situation exceptionnelle à Saint-Domingue, au XVIIIe siècle. Il en a été de même au temps de toutes les guerres maritimes un peu longues, lors des deux guerres de sept ans, de 1778 à 1782. Par le développement de l'absentéisme et du système complémentaire des procureurs tout-puissants, les plantations échappent de plus en plus aux propriétaires.

Parallèlement au mouvement d'autonomie politique, se poursuit a l'intérieur des plantations, un mouvement beaucoup plus fort et plus profond d'autonomie économique Le propriétaire n'est plus qu'un rentier, sans pouvoir, un porteur d'obligations qui n'a qu'une 
place restreinte dans le conseil de direction. Les plantations, en mille, cas deviennent des biens sans maîtres réels, directement intéressés à leur vie, risquant tout pour la bonne discipline et le bon esprit du travail. Cette rupture explique assez bien le caractère si vite irrémédiable de l'insurrection des esclaves qui fut désastreuse d'abord parce qu'elle fit le vide des blancs, des procureurs blancs.

Et puisqu'il s'agit d'exploitation agricole, ce sont les problèmes agricoles que ces documents éclaireront. Les Terrien insistent dans toutes leurs lettres sur la nécessité des fumures. C'était une assez grande nouveauté à Saint-Domingue que d'employer tant de fumier. C'était une nouveauté plus particulièrement sur les caféières, plantations établies en des quartiers neufs et généralement pourvus de grandes réserves de terres vierges. Ces terres récemment ouvertes aux cultures donnent donc déjà ces signes de fatigue. Il ne le semble pas ici. C'est l'application systématique d'idées d'agronomes, l'intention de conserver le plus longtemps possible les vertus fertilisantes d'un sol encore en plein rapport. Mais cet emploi méthodique très élargi des fumures suppose une organisation nouvelle du bétail, au moins sa demi-stabulation nocturne, des hangards, des mangeoires, donc une association mieux réglée, plus intime de la vie du bétail au travail général de la plantation. Le troupeau des mulets, chevaux et bœufs qui, sur les caféières n'avaient à fournir jusque là qu'un travail de transport, deviennent véritablement le cheptel, un élément de stabilisation des cultures. Les abatis quelque peu désordonnés des bois-debout, les défrichés iténérants vont être transformés par ces fumures intensives en véritables "jardins", en champs. L'homme a fixé sa conquête, la prise du sol est achevée. Le quartier de zone pionnière est devenu pour Saint-Domingue un vieux quartier.

Enfin, quand la perte de leur habitation a réduit leur fortune, on voit s'opérer pour la famille Navailles-Bonnas ce rétrécissement d'horizon économique et social qui fut une des conséquences les plus durables bien qu'unè des moins apparentes de la Révolution.

En 1789, les Navailles-Bonnas sont installés à Bordeaux, dans une vie active de relations, au milieu du monde parlementaire, au milieu du monde du grand commerce, au milieu du monde des propriétaires de Saint-Domingue. Et ils ont un œil sur leurs biens ruraux du pays de Bigorre. Leur fortune est partie en Bigorre, partie à Bor- 
deaux, partie à Saint-Domingue. Tous ces revenus leur permettent d'avoir un hôtel dans la grande capitale régionale, de tenir un assez grand train. Leurs plantations perdues les replient sur leurs terres, à la campagne. Les voilà uniquement propriétaires terriens, plus que jamais seigneurs de village. Plus d'hôtel en ville, plus de laquais frisés, mais un toit et une vie rustiques. L'on devient gentilhomme, exclusivement attaché maintenant aux étroits revenus des fermes. On vit du pays et on s'y attache, si bien que quand l'on parlera de l'indemnité de Saint-Domingue, ce ne sera plus pour cette famille désormais toute à sa petite vie rurale, qu'une aubaine inespérée, qui laissera regrets et déceptions...

Ce que l'émigration et le retour au milieu des biens nationaux vendus a produit chez tant d'autres, la perte de Saint-Domingue l'a fait chez les Navailles-Bonnas. Ils ont provoqué pour près d'un demi-siècle cet enracinement de la petite noblesse et ont préparé son influence politique et sociale, si grande dans certaines régions de la France rurale du XIXe siècle.

Refermons maintenant ce dossier de plantation de SaintDomingue et notons que chaque fois que l'on tente à travers un dossier de plantation de retrouver les grandes lignes de l'histoire de Saint-Domingue, la recherche paraît s'égarer. On suit une piste que coupent les lacunes des documents. Mais il en est presque toujours ainsi. Il n'est pas de dossier de plantation où soient alignés assez nombreux pour faire suite et ensemble, titres d'acquisition, procèsverbaux d'arpentage, concessions, comptes, correspondances, plans et journaux de travaux. Nous n'avons que des bornes irrégulières sur notre chemin. L'examen avance en zigzags. Mais tout n'est pas à regretter en cela. On ne touche pas ainsi à toutes les questions. Mais on en aborde de très variées; et l'on en éclaire mieux parfois l'importance et les connexités. C'est un éclairage qui semble plutôt dirigé vers les faits ordinaires, vers les réalités quotidiennes surprises dans le hasard de quelques documents qui survivent.

Ces documents n'apportent que de simples traits, quelques traits d'une histoire; mais ce sont des éléments positifs qui nous permettent d'imaginer nettement le cadre d'un passé déjà si lointain socialement.

Déplier le plus soigneusement possible ces documents, s'efforcer de résoudre ces difficultés et ces problèmes par une observation mi- 
nutieuse, au ras de terre, interroger sans cesse, voilà quelle a été notre ambition. Tout document, surtout fragmentaire est un merveilleux point de départ pour réfléchir, pour revenir et chercher encore. On s'est tracé un itinéraire d'étude. On a voulu montrer par cette analyse de début qu'une maison ne commence pas par le toit, que l'histoire d'une plantation complète l'histoire d'une autre plantation, l'explique.

Ce dossier est fragmentaire. C'est le malheur de tous les dossiers du monde et c'est par la comparaison méthodique de ces dossiers dépouillés avec ordre qu'on aura une vue générale à peu près exacte de l'évolution agricole, sociale et politique de la colonie.

Roger Massio, Professeur, Collège Vic-Bigorre, Hautes-Pyrenees, France. 